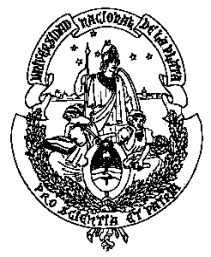

Maestría en Economía

Facultad de Ciencias Económicas

Universidad Nacional de La Plata

TESIS DE MAESTRIA

ALUMNO

Maria Victoria Fazio

TITULO

Incidencia de las Horas Trabajadas en el Rendimiento Académico de

Estudiantes Universitarios Argentinos

DIRECTOR

Alberto Porto

FECHA DE DEFENSA

$4 / 20 / 2004$ 
Universidad Nacional de La Plata
Maestría en Economía

\title{
Incidencia de las horas trabajadas en el rendimiento académico de estudiantes universitarios argentinos*
}

\author{
María Victoria Fazio
}

Director: Alberto Porto

Marzo 2004.

Resumen

En este trabajo se investiga la incidencia del trabajo del estudiante en su desempeño académico. Para analizar la relación entre el tiempo dedicado al trabajo y el desempeño académico de los alumnos se utilizan datos del Primer Censo de estudiantes de universidades nacionales de 1994. Los resultados compatibilizan con los argumentos contrapuestos sobre esta relación. Por un lado, si se postula un modelo lineal, se encuentra que las horas trabajadas inciden negativamente sobre el rendimiento. Por otro lado, en una especificación no lineal, las horas trabajadas se asocian en forma positiva y decreciente. En otros términos, para un nivel moderado de horas trabajadas, la incidencia puede ser positiva. Si se consideran sólo los alumnos con trabajos vinculados a la carrera, también se encuentra una relación no lineal, donde la relación positiva se evidencia para un mayor nivel de horas trabajadas. En contraste, si se analiza sólo el grupo con trabajos no vinculados, la relación resulta negativa, cualquiera sea el nivel de horas trabajadas. La investigación provee fundamentos empíricos a la legislación universitaria sobre pasantías acerca de las condiciones del trabajo del estudiante que preserven su desempeño académico.

Clasificación JE L: I21, I28, J31.

Palabras clave: trabajo del estudiante, rendimiento académico, horas trabajadas

\footnotetext{
* Q uiero agradecer muy especialmente al Dr. Alberto Porto por toda su dedicación y guía en el proceso de este trabajo. También agradezco los valiosos comentarios del lector Leonardo Gasparini e interacciones en etapas previas con un grupo de trabajo sobre "Rendimiento de los Estudiantes Universitarios y sus Determinantes" integrado por Luciano Di G resia, Alberto Porto, Laura Ripani y Walter Sosa Escudero. Valen las aclaraciones de responsabilidad del autor.

^vifazio@way.com.ar
} 


\section{Índice}

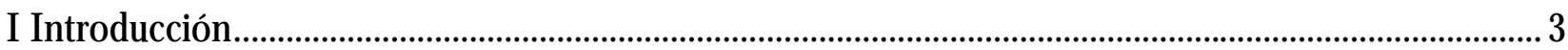

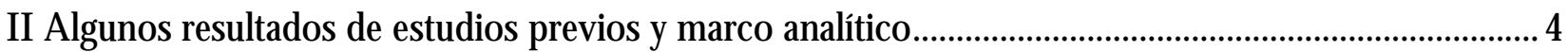

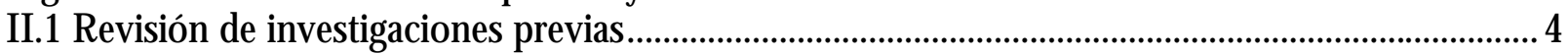

II.2 Marco analítico: enfoque de la función de producción educativa.................................................... 7

II.2.1 El enfoque de la función de producción del proceso educativo ................................................. 7

II.2.2 Medidas de producto del proceso educativo en términos de rendimiento académico .......... 8

III Principales características de los estudiantes universitarios según su condición laboral ..................... 9

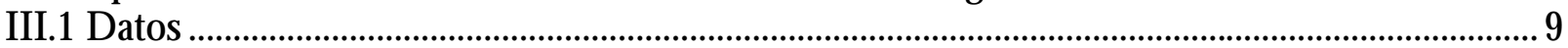

III.2 Características de los estudiantes según trabajen 0 no .............................................................10

III.3 Características de los estudiantes según su dedicación laboral por quintiles de rendimiento ..11

III.4 Análisis de las características de los alumnos que trabajan por deciles de horas trabajadas..... 13

III.4.1 Características de los alumnos que trabajan por deciles de horas trabajadas según la

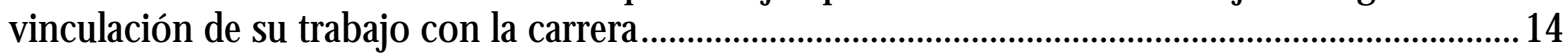

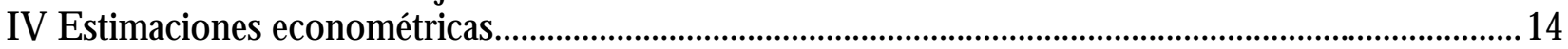

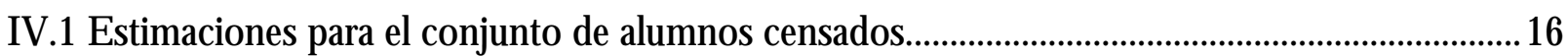

IV.2 Estimaciones para el grupo de alumnos que trabaja.......................................................................20

IV.2.1 A nálisis de la especificación lineal de la relación horas trabajadas- rendimiento.................20

IV .2.2 Análisis de la especificación no lineal de la relación horas trabajadas- rendimiento ...........23

V Conclusiones y líneas de investigación futura.........................................................................................25

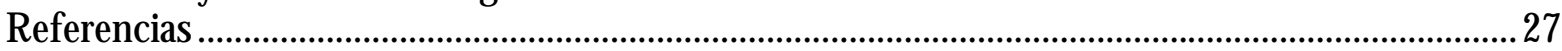

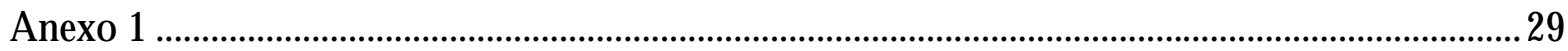

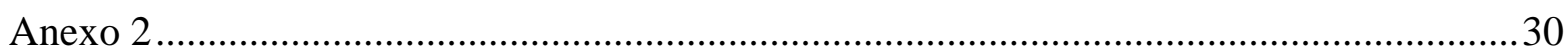




\section{Introducción}

Los costos y beneficios del trabajo del estudiante han sido analizados en términos de sus logros profesionales como graduado y sus logros académicos como alumno.

En términos de logros profesionales, en general, existe consenso entre los estudios empíricos acerca de que el trabajo del estudiante es beneficioso para la etapa laboral como graduado. Por ejemplo, se argumenta que otorga experiencia y conocimientos que aumentan la productividad futura. ${ }^{1}$ So bre todo si el trabajo brinda conocimientos complementarios a los de la carrera. ${ }^{2}$

En contraste, sobre los efectos del trabajo del estudiante en sus logros académicos como alumno existen argumentos contrapuestos en la literatura empírica.

Uno de ellos plantea que el trabajo incide negativamente sobre el desempeño del estudiante dado que, pensando el problema en términos de un modelo de suma cero, cuando emplea parte de su dotación de tiempo en trabajar, resta horas disponibles para desarrollar tareas como estudiar que mejoran su rendimiento académico. (Coleman, 1961).

En contraposición, también se argumenta que el trabajo del estudiante puede ser positivo por dos motivos principales. Una explicación surge de la idea de learning by doing o aprender haciendo, en este sentido, se señala que el trabajo del estudiante puede favorecer la transferencia de conocimientos relacionados con los temas de estudio. Por otra parte, aún cuando el trabajo no esté relacionado con la currícula, se sostiene que éste contribuye a disciplinar y refuerza el sentido de responsabilidad con efectos positivos sobre el desempeño académico. (Holland y Andre, 1987). Lo cual sugiere que los alumnos que trabajan quizás asignen de un modo más eficiente el tiempo.

Los datos del primer Censo de estudiantes de universidades nacionales revelan que más del $55 \%$ de los estudiantes universitarios no se dedican exclusivamente a estudiar, sino que además trabajan. También es significativo el porcentaje que trabaja más de 35 horas semanales (24\%). En promedio, asignan 31 horas por semana a su trabajo. ${ }^{3}$ A simismo, se observa que muy pocos alumnos se gradúan en el tiempo teórico de duración de la carrera. Para 20 carreras de todas las universidades públicas la duración media de la carrera supera en un $60 \%$ a la duración teórica. ${ }^{4}$

El objetivo principal de este trabajo consiste en investigar de qué manera las horas trabajadas por los estudiantes inciden en su desempeño académico.

Ante las contrapuestas versiones sobre el tema, en esta investigación se plantea una secuencia de preguntas empíricas sobre la relación entre el trabajo del estudiante y su desempeño 0 rendimiento académico. i) Una primera pregunta es cómo se refleja en el rendimiento el hecho de que un alumno trabaje. ii) Pero dado que los alumnos trabajan con distinta intensidad, es interesante preguntarse asimismo cómo se relacionan las horas trabajadas con el rendimiento académico. iii) Compatibilizando con los dos argumentos contrapuestos, podría esperarse que las horas trabajadas se relacionen positivamente con el rendimiento del alumno sólo en bajos niveles de horas trabajadas, pero que a más horas de trabajo, el alumno deba ajustar las horas de estudio, perjudicando su rendimiento. De modo que vale la pena indagar también si la incidencia de las horas trabajadas sobre el rendimiento se mantiene constante para cualquier cantidad de horas trabajadas (si la relación es lineal), o si depende del número de horas trabajadas (si es no lineal). iv) Por último, en todos los

\footnotetext{
${ }^{1}$ Las revisiones de Ruhm (1997) y Carr et al. (1996) encuentran en los estudios previos asociaciones positivas del trabajo del estudiante con sus ingresos futuros y la probabilidad de estar empleado.

2 Este argumento está presente en la Ley 25165/ 99 de Pasantías, por ejemplo en el Art.3, uno de los objetivos de la pasantía es "Formar al estudiante en aspectos que le serán de utilidad en su posterior búsqueda laboral".

${ }^{3}$ En base a datos del Censo de Estudiantes de Universidades Nacionales de 1994. Secretaría de Políticas Universitarias.

4 A partir del ratio de duración media de la carrera/ duración teórica para 1999. (Capítulo 3 del Anuario 99-00 de Estadísticas Universitarias, Secretaría de Políticas Universitarias).

${ }^{5} \mathrm{G}$ ertel (1999) atribuye este retraso, entre otros factores, al alto porcentaje de estudiantes que trabaja.
} 
casos, cómo incide además en la relación trabajo-rendimiento el hecho de que el trabajo esté vinculado 0 no con la carrera que cursa el estudiante. ${ }^{6}$

Para analizar empíricamente estas preguntas, se utilizó información que surge del Primer Censo de estudiantes de universidades nacionales de 1994, que relevó datos sobre la distribución y características académicas y sociales de los alumnos inscriptos en carreras universitarias de grado.

El estudio de la relación trabajo-rendimiento académico tiene relevancia práctica por sus conclusiones acerca de las condiciones que debería reunir una experiencia laboral para preservar el desempeño académico del alumno. En este sentido, a partir del vínculo de las conclusiones con las regulaciones de las prácticas laborales que establece la Ley de Pasantías, podría decirse que los resultados de la investigación aparecen como una fundamentación empírica de esas normas.

También tiene implicancias en términos de igualdad de oportunidades, uno de los principios que sustenta la legislación universitaria. Por ejemplo, si se encontrara un efecto negativo del trabajo del estudiante sobre su desempeño, los alumnos con necesidades económicas tales que necesiten trabajar para cubrir sus estudios, estarían en desventaja respecto a quienes se financian por otros medios. En este sentido, las conclusiones acerca de esta relación serán también relevantes en términos de políticas de financiamiento del alumno a través del otorgamiento de becas, créditos 0 pasantías, entre otras.

El trabajo se organiza en cinco secciones principales. En la que sigue, se analiza la literatura previa sobre la relación entre el trabajo y los logros académicos del estudiante y se describe el marco analítico en el que se basarán las predicciones de los modelos econométricos planteados más adelante. En la sección III se describen las características del grupo de estudiantes universitarios encuestado en el censo de 1994 que se diferencian por su condición y dedicación laboral. En la sección IV se estiman por métodos econométricos los modelos de rendimiento postulados y, por último, se resumen los resultados y se presentan las conclusiones en la sección V.

\section{Algunos resultados de estudios previos y marco analítico}

\section{II.1 Revisión de investigaciones previas}

La incidencia del trabajo del estudiante en sus logros académicos y profesionales ha sido analizada en trabajos empíricos que difieren sustancialmente en términos de las muestras, los métodos econométricos que utilizan y la elección de la variable dependiente. En cuanto a sus conclusiones, en el caso de los trabajos sobre la relación trabajo del estudiante-logros profesionales, luego de graduados, en general se argumenta que el trabajo del estudiante es una práctica favorable en la preparación para la etapa laboral posterior. ${ }^{7}$

En cambio, del análisis de la literatura sobre la relación trabajo del estudiante-logros académicos se extraen conclusiones ambiguas.

\footnotetext{
${ }^{6}$ Un ejemplo de trabajo vinculado podrían ser las pasantías. En el Art. 2 de la Ley 25165 de Pasantías se establece que "Se entenderá como "pasantía" a la extensión orgánica del sistema educativo en el ámbito de empresas u organismos públicos o privados, en los cuales los alumnos realizarán residencias programadas u otras formas de prácticas supervisadas relacionadas con su formación y especialización, llevadas a cabo bajo la organización y control de las unidades educativas que lo integran y a las que aquellos pertenecen...". En el Art.3, establece que uno de los objetivos del sistema de pasantías es "Brindar experiencia práctica complementaria de la formación teórica elegida que habilite para el ejercicio de la profesión u oficio"

${ }^{7}$ Por ejemplo, Ruhm (1997), utilizando una base que sigue a cohortes de estudiantes desde la escuela secundaria hasta sus primeras experiencias laborales como graduados, encuentra que trabajar una cantidad moderada de horas durante el curso de los estudios se relaciona positivamente con los ingresos futuros y la categoría ocupacional, al menos en los primeros años luego de graduarse en el secundario.
} 
Por un lado, los trabajos que argumentan que las horas trabajadas impactan negativamente en el desempeño o rendimiento académico del alumno, se basan en un modelo de suma cero, donde el tiempo invertido en el trabajo es tiempo que se extrae al desarrollo de actividades que mejoran el desempeño como el estudio. (Coleman, 1961). Ejemplos de trabajos que encuentran una relación negativa son Tyler (2003), Stinebrickner y Stinebrickner (2003), Paul (1982), Eckstein y Wolpin (1997), entre otros.

En contraste, también se argumenta que trabajar, particularmente en un trabajo complementario al tema de estudio, puede tener un efecto positivo en el desempeño académico. Este argumento se basa en la idea de learning by doing, que señala que los conocimientos se fijan más traduciéndolos a la práctica. A simismo, resaltan que el trabajo en general, enseña y refuerza el sentido de responsabilidad al alumno que trabaja y que, a través de este mejor comportamiento, mejoran sus resultados académicos. (Holland y Andre, 1987). Ejemplos en los que se encuentran estos signos son Schill et al. (1985), Lillydahl (1990), Turner (1994), D 'Amico (1984).

Resaltando este contraste de conclusiones, Ruhm (1997) en una revisión muy completa de la investigación de distintas disciplinas que analizaron el efecto del trabajo en los logros del estudiante, concluye que no hay consenso sobre si el trabajo del estudiante mejora o empeora su rendimiento académico. Y señala que son pocos los trabajos que han hecho esfuerzos para considerar la posible determinación simultánea (o endogeneidad) entre las variables relacionadas a la decisión de trabajar y la variable de rendimiento. ${ }^{8}$ Como plantean Stinebrickner et al. (2003), en muchos casos, esto se debe a que controlar este problema implica el uso del método de Variables instrumentales, cuya aplicación exitosa depende de contar con una variable que sirva como instrumento. ${ }^{9}$

En este sentido, de acuerdo con Tyler (2003), las estimaciones que por Mínimos Cuadrados Ordinarios (MCO) reflejan un impacto positivo del trabajo sobre el rendimiento, subestiman el efecto negativo del trabajo sobre el desempeño.

Por ejemplo, las estimaciones presentadas en Turner (1994) para estudiantes de educación secundaria y postsecundaria en Estados Unidos por el método de Mínimos cuadrados, muestra que trabajar un número moderado de horas resultaría potencialmente beneficioso para el desempeño del alumno. Sin embargo, al intentar aplicar el método de Variables instrumentales, cuenta con instrumentos no significativos.

En contraste, un estudio que constituye un buen ejemplo en la aplicación del método de Variables instrumentales es el trabajo de Stinebrickner et al.(2003). Encuentran que una hora adicional de trabajo del estudiante impacta negativamente sobre su rendimiento académico. Para ello cuentan con una muestra única de una universidad de Canadá que incluye programas de empleo en su currícula y la disponibilidad de información les permite trabajar con el método de Variables instrumentales utilizando las horas trabajadas por los ingresantes como un instrumento razonable para controlar el problema de endogeneidad.

Tyler (2003) encuentra efectos negativos de las horas trabajadas sobre el rendimiento académico tanto por MCO como por Variables instrumentales, sólo que los efectos son más grandes en este último caso.

Los trabajos anteriormente citados plantean una relación lineal entre las horas trabajadas y el rendimiento académico, sin embargo, puede ser cierto que la relación varíe dependiendo de la cantidad de horas que el estudiante trabaje. En este sentido, el trabajo de Lillydahl (1990) encuentra

\footnotetext{
${ }^{8}$ Se advierte sobre endogeneidad porque la relación puede reflejar la causalidad contraria. Por ejemplo, se puede dar una relación causal en la que los alumnos de alto rendimiento, tienen más alta motivación y por consiguiente esto los hace más proclives a involucrarse en el trabajo. Como consecuencia, dado que la motivación es difícilmente observable, en los modelos de regresión simple que no puedan incluir la motivación, parte de la variación en el rendimiento que no pueda atribuirse a la variación en la motivación puede ser erróneamente atribuida a diferencias en la condición laboral. (Stinebrickner et al., 2003).

${ }_{9}$ Un instrumento para este ejemplo sería una variable que se encuentre correlacionada con las horas trabajadas, pero que sólo se encuentre correlacionada con el rendimiento a través del efecto que ejerce sobre el mismo y no a la inversa.
} 
un efecto no lineal entre las horas trabajadas en el rendimiento medido como el GPA (Grade point average) estimando por Mínimos cuadrados en dos etapas. ${ }^{10}$ En otros términos, el efecto es positivo hasta las 13.5 horas de trabajo por semana y se torna negativo más allá de esa cantidad de horas trabajadas. O tro estudio que encuentra una relación curvilínea entre las horas trabajadas y el GPA es el de Schill et al. (1985), quienes documentan que los estudiantes que trabajaban hasta 20 horas semanales evidenciaron los más altos promedios. Asimismo, Hood et al. (1992) encuentran que los GPA son más altos entre estudiantes que trabajan moderadas cantidades de horas (entre 7 a 14 horas semanales).

O tro resultado interesante documentado en la literatura previa es que en el análisis de la relación trabajo-rendimiento es importante considerar el grado de vinculación del trabajo con los temas de estudio. Por ejemplo, Stern et al. (1997) encuentran que el efecto adverso de las horas trabajadas sobre el rendimiento es menor cuando el trabajo se vincula con la actividad curricular. En este sentido, Pauly et al. (1995) señalan que una estrategia para reducir los efectos negativos del trabajo es que existan vínculos más explícitos entre la actividad académica y su actividad laboral. Asimismo, Ehrenberg y Sherman (1987) encuentran por un lado efectos positivos del trabajo para empleos dentro del campus universitario, pero negativos en empleos fuera del campus.

Una de las motivaciones de este trabajo es que la mayoría de los estudios comentados presenta evidencia sólo para Estados Unidos, donde la preocupación por la creciente participación de los estudiantes (principalmente secundarios) en el mercado laboral, acompañada de bajos niveles de desempeño en relación con los de otros países, ha generado muchas investigaciones acerca de la relación entre el trabajo y el rendimineto académico del estudiante. ${ }^{11}$

Para Argentina, no se encuentran trabajos específicos sobre el tema planteado. Entre las evidencias disponibles, el estudio de Di Gresia, Porto, Ripani y Sosa Escudero (2003) provee un exhaustivo análisis de los factores determinantes del rendimiento académico de los estudiantes de las universidades públicas argentinas. Para ello, utilizan la misma base de datos que en el presente trabajo proveniente del Censo de estudiantes de universidades nacionales de 1994 . Uno de los resultados encontrados para el total de universidades, refleja que el alumno que trabaja tendría un rendimiento superior respecto al que no trabaja, medido en términos de cantidad de materias aprobadas por año.

En contraste, en otro estudio sobre determinantes del rendimiento educativo, Di Gresia y Porto (2004) encuentran una relación negativa entre las horas trabajadas y el rendimiento académico, medido también por la cantidad de materias por año. En este caso, el estudio controla por las potenciales heterogeneidades entre cohortes y carreras y por la autoselección de los alumnos entre carreras, dado que se sigue solamente a la cohorte de los alumnos que ingresaron en el año 2000 a la Facultad de Ciencias E conómicas hasta la etapa de finalización del ciclo básico de materias.

Quizás a favor de una relación negativa entre el trabajo del estudiante y su rendimiento se encuentran los trabajos relacionados con la probabilidad de deserción universitaria, como el de Giovagnoli (2002), que encuentra que un alumno que inicia sus estudios trabajando tiene 3.4 veces más riesgo de abandono que quienes no tienen responsabilidad laboral.

En general, en los trabajos que encuentran una relación trabajo-rendimiento positiva, los datos sugieren que los efectos positivos del trabajo del estudiante en sus rendimientos se maximizan en niveles bajos o intermedios de horas trabajadas y que dependen del tipo de trabajo y su vinculación con la carrera. Mientras que en niveles altos de horas trabajadas el efecto de trabajar es negativo sobre los rendimientos académicos.

\footnotetext{
${ }^{10}$ El GPA (Grade Point Average) es una medida análoga al promedio académico en Argentina, pero con ajuste por los métodos de corrección de exámenes de las distintas carreras.

${ }^{11}$ D e hecho, la creciente cantidad de horas trabajadas ha llevado a legislar a algunos estados la reducción del tiempo máximo en el trabajo. Por ejemplo, en Massachusetts, de 45 horas a 28 horas por semana. (Tyler, 2003).
} 


\section{II.2 Marco analítico: enfoque de la función de producción educativa}

Los trabajos comentados que analizan la pregunta empírica planteada sobre la relación entre el trabajo y el desempeño académico del estudiante se circunscriben en la literatura de la Economía de la educación que estudia los factores determinantes de los logros académicos de los alumnos. El propósito de esta parte del trabajo consiste en proveer el marco analítico utilizado en el estudio de estos determinantes desde un enfoque económico.

\section{II.2.1 El enfoque de la función de producción del proceso educativo}

El marco analítico que se utiliza en los trabajos empíricos sobre los determinantes de los logros de los estudiantes desde una perspectiva económica consiste en el enfoque de la función de producción del proceso educativo. El modelo que plantea este enfoque se basa en la teoría microeconómica de la firma para intentar explicar el "producto" del proceso educativo en función de los "insumos" que utiliza. Entre los primeros trabajos acerca de las funciones de producción educativa se destaca el informe de Coleman et al. (1966) para Estados Unidos, a partir del cual se creó una controversia sobre la conclusión de que las escuelas no son tan importantes en afectar los logros de los alumnos, sino que las familias y el grupo de pares son los determinantes primarios de las variaciones en el desempeño académico. Los trabajos de Hanushek (1993), Pritchett y Filmer (1997) y Llach et al. (2000) son referencias que desarrollan un análisis abarcativo de este enfoque.

La definición de la variable dependiente entendida como el "producto" desde la perspectiva de producción educativa difiere según los trabajos empíricos. Los estudios aproximan esta variable a través de distintos indicadores, ya que en el proceso educativo se generan múltiples productos.

Entre las alternativas utilizadas para medir la producción del proceso educativo se encuentran: el valor presente neto de los ingresos futuros de los estudiantes; la elección sobre el modo de vida (por ejemplo, el estado civil, el tener hijos); el esfuerzo realizado por los estudiantes (por ejemplo, las horas de estudio, la asistencia a clases); y los logros académicos.

Este trabajo se vincula con el estudio de este último tipo de "producto" generado por el proceso educativo, los logros académicos de los estudiantes en su etapa de alumno universitario. Estos pueden a su vez definirse según distintas medidas de resultado: como la duración de la carrera, el número de materias aprobadas en promedio por año, el promedio de notas, el resultado de evaluaciones estandarizadas sobre el nivel de conocimiento en materias como lengua y matemática, entre otras aproximaciones.

Para este estudio se adopta como medida de logro académico del estudiante al número de materias que lleva aprobadas por año desde el ingreso a la facultad. Sobre esta medida de productividad se desarrollará un análisis más adelante.

La segunda parte en la definición del modelo de producción del proceso educativo es la indentificación de los factores explicativos o "insumos" de la medida de "producto" que se utilice. En esta etapa la principal complejidad se encuentra en que los factores propios de los actores principales del proceso educativo, tales como la motivación del estudiante, sus habilidades innatas, 0 la calidad del docente son difíciles de medir. Estas restricciones se reflejan en que la mayoría de los estudios empíricos dejan sin explicar un alto porcentaje de la variación en la variable dependiente analizada.

Las variables que por lo general se utilizan en los modelos para explicar el rendimiento académico del alumno, se pueden categorizar en cinco grupos:

$\mathrm{V}$ ariables relacionadas con la persona: dentro de este grupo se ha encontrado que el sexo, la edad, las habilidades innatas (difícilmente medibles), el esfuerzo medido en términos de horas de estudio, el trabajo y las horas trabajadas son factores significativos para explicar el rendimiento académico.

$\mathrm{V}$ ariables del entorno familiar del estudiante: por lo general han resultado significativas variables como la educación de los padres, la ocupación de los padres y el nivel de ingreso familiar. De acuerdo con Hanushek (1986), el entorno familiar es claramente relevante en la explicación de las 
diferencias en los logros de los estudiantes. Independientemente de cómo se mida, los padres más educados y ricos tienen en promedio hijos con mayor performance educativa. Con respecto al tamaño de la familia, los efectos en general resultan ambiguos (Summers y Wolfe (1977)).

$\mathrm{V}$ ariables relacionadas on los rearsos docentes y de equipamiento y factores institucionales: como la relación entre el número de docentes y alumnos, el gasto en insumos educativos por alumno, el ambiente académico (disponibilidad de recursos como bibliotecas, aulas, etc.), entre otros. Sobre el efecto de estos recursos existen resultados ambiguos. Por ejemplo, el informe de Coleman et al. (1966) para Estados Unidos encuentra que tienen una incidencia menor, mientras que McG uckin y Winkler (1979), al introducirlos en el modelo como el uso que los estudiantes hacen de los recursos, concluyen que son factores que influyen positivamente en la producción de educación. En contraste, Hanushek (1986) señala que de 112 estudios sobre esa relación, sólo en 9 el signo es positivo y significativo. En un trabajo reciente, Hanushek (2003) argumenta que la explicación a estos efectos encontrados proviene de que la asignación de recursos no se basa en incentivos.

$V$ ariables regionales: este grupo de variables se incluyen en los modelos para determinar si existe un efecto específico de la región de residencia que no resulte captado por los distintos aspectos del entomo económico y social familiar. De acuerdo con Llach et al (2000), el efecto del lugar de residencia puede explicarse por las externalidades que se generan por el capital humano específico que contribuye a la concentración de actividades en un determinado espacio. Por ejemplo, es de esperar que donde haya más facultades haya mayor cantidad de buenos profesores y, por lo tanto, que los alumnos que asistan a esa facultad se beneficien de un efecto específico de la región.

En resumen, en estudios realizados para distintas muestras se han encontrado estadísticamente signficativas variables de estos cuatro grupos. Vale aclarar que de acuerdo a la pregunta empírica que se planteen 0 a la disponibilidad de información, las variables explicativas utilizadas y las metodologías de estimación varían entre los trabajos. Así como también difieren en las medidas del producto del proceso educativo que utilizan, como se explicara anteriormente. Sobre la medida de rendimiento académico utilizada en este trabajo en comparación con otros indicadores se hará un breve comentario en el siguiente punto .

\section{II.2.2 Medidas de producto del proceso educativo en términos de rendimiento académico}

La definición de un indicador para medir el desempeño de los estudiantes de cualquier nivel educativo difiere entre las investigaciones que siguen el enfoque de la función de producción del proceso educativo. Ejemplos de las medidas utilizadas son el promedio de notas en materias aprobadas 0 rendidas, los resultados de tests estandarizados de aptitudes en lengua o matemática, la probabilidad de deserción, el ranking de alumnos en la clase, entre otras. En cuanto al promedio de notas, se considera que ceteris paribus otras condiciones, el alumno que obtiene una mayor nota al evaluarlo, incorporó más capital humano. Un planteo que suele realizarse a esta medida es que puede estar sujeta a la varianza en la corrección de exámenes entre universidades, carreras o departamentos. No obstante, la mayoría de los trabajos, en particular los que estudian la relación entre el trabajo y el rendimiento académico, se refieren a esta medida de desempeño y además es contemplada en los requisitos de becas, promoción de materias y empleos como principal indicador de los resultados académicos del alumno.

En el Censo de estudiantes de universidades nacionales de 1994 que se utiliza como fuente de información en este trabajo no se cuenta con este tipo de medida de rendimiento. En su lugar, presenta como indicador del desempeño del estudiante, al número total de materias aprobadas desde el ingreso a la facultad. Con esta información, se adopta para esta investigación una medida de producto que corrige al total de materias aprobadas por el número de años desde aquel ingreso. En este sentido, se utiliza el número de materias aprobadas por año como indicador de la productividad media del estudiante, donde el producto total está representado por el total de materias aprobadas y el 
insumo es el número de años desde que estudiante ingresó a la facultad. ${ }^{12}$ En comparación con el promedio académico de notas, de acuerdo a este indicador tendrá mayor productividad media aquel alumno que haya aprobado una mayor cantidad promedio anual de materias, independientemente de si lo haya conseguido con más o menos nota. La noción de rendimiento intrínseca en esta medida es que el alumno que apruebe mayor cantidad de materias por año habrá adquirido al menos el mínimo capital humano requerido en menor tiempo. Y por consiguiente, no sólo será menor la probabilidad de que se vuelva obsoleto el capital ya incorporado, sino que también demorará menos en recuperar su inversión en el mercado laboral. En este sentido, la medida de rendimiento utilizada también se vincula con la demora en la graduación.

A simismo, sobre esta medida de desempeño se fundamentan reglamentos de la legislación universitaria. Por ejemplo, los requerimientos para la obtención del título, para establecer la condición de regularidad del alumno, para la adjudicación de becas, pasantías y otras políticas de financiamiento del alumno. ${ }^{13}$

\section{Principales características de los estudiantes universitarios según su condición laboral}

El análisis descriptivo del grupo de estudiantes universitarios según su condición laboral evidencia diferencias en las características sociales, económicas y académicas de los alumnos que trabajan respecto a los que no trabajan. Asimismo, se encuentra que estas diferencias son más notorias entre quienes trabajan, según las horas trabajadas y la vinculación de su trabajo con la carrera que cursan. En esta sección se analizan las tablas 3.1 a 3.4 que exponen estas evidencias obtenidas a partir del Censo de estudiantes de universidades nacionales de 1994.

\section{III.1 Datos}

La principal fuente de datos utilizada para el análisis empírico de este estudio es el Primer Censo de Estudiantes de Universidades Nacionales de 1994, realizado por el Consejo Interuniversitario Nacional (CIN) junto con la Secretaría de Políticas Universitarias (SPU). Si bien anteriormente algunas universidades han realizado censos con regularidad, este es el primer relevamiento con cobertura nacional. El censo fue de cumplimiento obligatorio para todos los alumnos inscriptos a la fecha de realización de la encuesta en una carrera universitaria de grado. Cuenta con 615.796 observaciones de alumnos distribuidos en 31 universidades nacionales. ${ }^{14} \mathrm{El}$ objetivo de este relevamiento consistió en recoger y compilar información actualizada y confiable sobre la cantidad y distribución de alumnos, el tipo y grado de avance en la carrera que cursan y sus características sociales y demográficas.

En particular, para los objetivos de este trabajo cuenta con información apropiada sobre las condiciones laborales del estudiante.

\footnotetext{
12 Esta medida es implementada en el trabajo de Di G resia et al. (2003).

13 En el Art.50 de la Ley 24521/95 de Educación Superior se establece que las normas de regularidad que dicten las universidades deben fijar el mínimo rendimiento académico exigible, debiendo preverse que los alumnos aprueben por lo menos dos materias por año, salvo cuando el plan de estudios prevea menos de cuatro materias, en cuyo caso deben aprobar una como mínimo. Por otra parte, el Reglamento General de Becas del Ministerio de Educación de la Nación establece que, para aspirar a la beca, además de tener un promedio no inferior a 7 puntos con aplazos, los alumnos deben cumplir con el mínimo de materias que exige la Ley de Educación Superior para ser alumno regular.

${ }^{14} \mathrm{D}$ e acuerdo con el Anuario 1998 de Estadísticas Universitarias en Argentina hay un total de 36 universidades públicas. Las facultades suman 199 y en ellas se distribuyen 899 carreras de grado.
} 


\section{III.2 Caracteństicas de los estudiantes según trabajen o no}

Los alumnos difieren en su rendimiento académico y estudiar la relación de esas diferencias con la condición laboral del estudiante, entre otros factores explicativos, es uno de los objetivos principales de este trabajo. Para describir comparativamente al grupo de estudiantes que trabaja respecto al que no trabaja, se analizaron una serie de características sociales, económicas y académicas.

En la tabla 3.1 se exponen algunas características de los alumnos censados según su condición de actividad. En primer lugar, más del 55\% de los alumnos universitarios encuestados trabajan. Respecto al grupo que no trabaja, en el que trabaja se observa que es mayor la participación de los hombres (52.7\%), la edad promedio, la participación de casados y el porcentaje que ha ingresado más de una vez a la facultad.

Tabla 3.1 Características de los estudiantes universitarios según su condición laboral

\begin{tabular}{|c|c|c|c|}
\hline Condición laboral & Trabajan & No trabajan & Total \\
\hline Edad & 25.7 & 21.8 & 24.0 \\
\hline$\%$ Varones & 52.7 & 41.7 & 47.8 \\
\hline$\%$ Mujeres & 47.3 & 58.3 & 52.2 \\
\hline$\%$ Solteros & 75.8 & 91.3 & 82.7 \\
\hline$\%$ Casados & 19.2 & 7.0 & 13.8 \\
\hline \% Reingresantes & 4.8 & 3.3 & 4.1 \\
\hline Años desde ingreso & 5.6 & 4.1 & 4.9 \\
\hline Horas de estudio & 24.8 & 33.9 & 29.0 \\
\hline Rendimiento & 2.7 & 2.6 & 2.7 \\
\hline Horas trabajadas & $\overline{\overline{31.0}}$ & ……...... & …….... \\
\hline Se financian con salario (en \%) & 41.3 & .............. & \\
\hline Trabajo vinculado (en \%) & 43.1 & .............. & \\
\hline
\end{tabular}

Elaboración propia en base al Censo de estudiantes de universidades nacionales de 1994.

Es interesante notar que el promedio de horas de estudio incluyendo la asistencia a clases es casi 10 horas menor para el grupo que trabaja, comparado con el tiempo que estudian los alumnos que no trabajan. Paradójicamente, se encuentra que el rendimiento académico medido como el número de materias aprobadas por año es levemente superior para quienes trabajan. Sin embargo, como se comentará posteriormente, este resultado puede estar influenciado porque no discrimina por la intensidad del trabajo realizado. Esto es, en el grupo que trabaja se incluyen alumnos que trabajan muy pocas horas junto con los que dedican varias horas a trabajar y por eso el promedio puede no ser representativo del rendimiento de estos últimos. ${ }^{15}$ Como se verá, el grupo que trabaja también se distingue por la vinculación del trabajo con los contenidos de estudio.

En la tabla 3.1 también se presenta información sobre las horas trabajadas, la fuente de financiamiento de los estudios y la vinculación del trabajo con los estudios del grupo que trabaja. En primer lugar, en promedio los alumnos que trabajan dedican 31 horas semanales al trabajo. En cuanto a las características económicas del grupo que trabaja, el salario es la única fuente que les provee ingresos para financiar sus estudios a $41 \%$ de los alumnos que trabajan. Se entiende que conocer la vinculación del trabajo con la carrera de estudio es un dato importante para analizar la relación que tiene el trabajo con el rendimiento académico. La actividad que realizan es complementaria o está vinculada con los temas de estudio para el 43\% de los alumnos que trabajan.

En resumen, el análisis descriptivo de los grupos de alumnos que se distinguen por su condición de actividad revela que se diferencian también en todas las características analizadas. D el análisis anterior resulta que es más probable que el alumno que trabaja tenga una edad mayor que uno que no trabaja, que sea varón, que esté casado, que haya reingresado a la facultad, que estudie

${ }^{15}$ Asimismo, en el rendimiento del grupo que trabaja se observa mayor presencia de valores extremos. 
casi 10 horas menos por semana y sin embargo, que tenga un rendimiento levemente superior. No obstante, se observará que esta última evidencia no se mantiene para todos los alumnos que trabajan, ya que habrá que considerar cuántas horas trabajan y si el trabajo se vincula con los estudios.

En el análisis condicional de la sección IV se controlará por estas y otras características que difieren entre los alumnos que trabajan para estudiar la relación entre el trabajo del estudiante y su rendimiento académico. Pero antes, se continuará con una diferenciación más desagregada de la condición laboral de los alumnos.

\section{III.3 Caractenísticas de los estudiantes según su dedicación laboral por quintiles de rendimiento}

En contraste con la tabla anterior, en la tabla 3.2 se expone una distinción más desagregada entre tres tipos de alumnos por su condición de actividad según: no trabajen, trabajen tiempo parcial, o trabajen tiempo completo. El grupo de alumnos que trabajan tiempo completo se compone de los alumnos que trabajan 35 o más horas por semana. Mientras que el grupo de tiempo parcial trabaja menos de 35 horas semanales. ${ }^{16}$

A su vez, en la tabla se analiza el patrón de algunas características de los alumnos según su desempeño, por quintiles de rendimiento académico.

En primer lugar, en promedio por quintiles se observa que según la reclasificación por condición laboral, un $42 \%$ de alumnos no trabajan, un 34\% trabaja tiempo parcial y un $24 \%$ trabaja tiempo completo. Asimismo, de izquierda a derecha, se observa que la participación de los alumnos que no trabajan y de los que trabajan tiempo parcial es creciente por quintiles de rendimiento, mientras que la participación de los que trabajan tiempo completo decrece en los grupos de mayor rendimiento.

El grupo que trabaja tiempo completo tiene una edad promedio superior al resto. Asimismo, en ese grupo también es mayor la participación de varones, el porcentaje de casados y el tiempo de permanencia en la facultad. En cambio, por quintiles se observa que la edad promedio es levemente decreciente, también la participación de varones y de casados y el tiempo de permanencia en la facultad, para todos los grupos de dedicación laboral.

Si bien en términos de rendimiento no se observan grandes diferencias, puede verse que el resultado de la tabla 3.1 acerca de que el rendimiento es superior entre los alumnos que trabajan estaba influido por el rendimiento de los alumnos que trabajan tiempo parcial. Esto significa que no todos los alumnos que trabajan tienen un rendimiento mayor que los que no trabajan. Como puede verse, ese resultado se da sólo para los alumnos que trabajan pocas horas por semana y no para los que trabajan tiempo completo. Estas diferencias en rendimiento según la intensidad del trabajo del estudiante se analizarán en la sección de estimaciones econométricas y en la próxima tabla.

El análisis de las horas de estudio presenta resultados interesantes según el grupo de alumnos que se observe. Por un lado, se ve que las horas de estudio son menores cuantas más horas trabaje el alumno. Esto refuerza la idea de la correlación negativa entre horas de estudio y horas trabajadas, dada la restricción temporal del estudiante. Por quintiles, se observa que a mayor rendimiento, las horas de estudio decrecen para los alumnos que no trabajan y para los que trabajan tiempo parcial. Evidencia que sugiere que los alumnos de mayor rendimiento se benefician marginalmente menos con más horas de estudio. En cambio, para el grupo de tiempo completo, las horas de estudio aumentan por quintiles de rendimiento.

\footnotetext{
${ }^{16}$ Esta clasificación se corresponde con la definición de tiempo parcial según la mayoría de las regulaciones laborales en países de la OECD.
} 
Tabla 3.2 Características de los estudiantes según su dedicación laboral por quintiles de rendimiento

\begin{tabular}{|c|c|c|c|c|c|c|}
\hline & \multicolumn{6}{|c|}{ Quintiles de rendimiento académico } \\
\hline & 1 & 2 & 3 & 4 & 5 & Total \\
\hline Total & 100.0 & 100.0 & 100.0 & 100.0 & 100.0 & 100.0 \\
\hline No trabajan & 40.3 & 42.6 & 42.3 & 44.2 & 40.8 & 42.0 \\
\hline Trabajan tiempo parcial & 32.2 & 32.7 & 33.7 & 34.6 & 37.3 & 34.1 \\
\hline Trabajan tiempo completo & 27.5 & 24.8 & 24.1 & 21.3 & 21.9 & 23.9 \\
\hline Edad & 25.0 & 24.6 & 24.7 & 23.5 & 24.3 & 24.4 \\
\hline No trabajan & 22.7 & 22.6 & 22.9 & 22.1 & 22.8 & 22.6 \\
\hline Trabajan tiempo parcial & 25.6 & 25.1 & 25.0 & 23.8 & 24.5 & 24.8 \\
\hline Trabajan tiempo completo & 27.7 & 27.3 & 27.3 & 25.9 & 26.9 & 27.1 \\
\hline Varones (en\%) & 50.0 & 49.7 & 48.9 & 47.3 & 45.9 & $\overline{48.4}$ \\
\hline No trabajan & 42.6 & 42.5 & 42.5 & 41.6 & 41.3 & 42.1 \\
\hline Trabajan tiempo parcial & 47.9 & 48.0 & 46.8 & 46.0 & 43.5 & 46.3 \\
\hline Trabajan tiempo completo & 63.2 & 64.4 & 63.2 & 61.4 & 58.7 & 62.3 \\
\hline Casados (en\%) & 15.5 & 15.5 & 15.9 & 11.5 & 13.6 & 14.4 \\
\hline No trabajan & 8.9 & 8.6 & 9.1 & 6.9 & 8.5 & 8.3 \\
\hline Trabajan tiempo parcial & 16.1 & 16.0 & 15.8 & 11.3 & 13.4 & 14.4 \\
\hline Trabajan tiempo completo & 24.6 & 26.9 & 28.0 & 21.3 & 23.5 & 25.0 \\
\hline Años desde el ingreso & 5.3 & 5.2 & 5.5 & 4.4 & 4.0 & 4.9 \\
\hline No trabajan & 4.2 & 4.2 & 4.6 & 3.9 & 3.8 & 4.1 \\
\hline Trabajan tiempo parcial & 5.6 & 5.5 & 5.7 & 4.6 & 4.2 & 5.1 \\
\hline Trabajan tiempo completo & 6.7 & 6.6 & 6.7 & 5.2 & 4.2 & 6.0 \\
\hline Rendimiento & 0.8 & 1.7 & 2.4 & 3.2 & 5.2 & 2.7 \\
\hline No trabajan & 0.8 & 1.7 & 2.4 & 3.2 & 4.9 & 2.62 \\
\hline Trabajan tiempo parcial & 0.8 & 1.7 & 2.4 & 3.2 & 5.2 & 2.74 \\
\hline Trabajan tiempo completo & 0.8 & 1.7 & 2.4 & 3.2 & 5.5 & 2.60 \\
\hline Horas de estudio semanales & 26.5 & 28.5 & 29.5 & 30.7 & 30.5 & 29.2 \\
\hline No trabajan & 31.5 & 34.1 & 35.5 & 36.0 & 36.0 & 34.7 \\
\hline Trabajan tiempo parcial & 24.5 & 26.5 & 27.5 & 28.8 & 28.6 & 27.3 \\
\hline Trabajan tiempo completo & 20.8 & 21.0 & 21.2 & 22.6 & 23.1 & 21.7 \\
\hline Horas trabajadas semanales & 32.4 & 31.4 & 30.9 & 29.6 & 29.3 & 30.7 \\
\hline Trabajan tiempo parcial & 20.7 & 20.6 & 20.4 & 20.2 & 20.1 & 20.4 \\
\hline Trabajan tiempo completo & 46.0 & 45.7 & 45.5 & 44.9 & 44.8 & 45.4 \\
\hline Trabajo vinculado (en \%) & 35.3 & 40.1 & 46.2 & 48.3 & 56.2 & 45.3 \\
\hline Trabajan tiempo parcial & 31.6 & 36.9 & 43.1 & 45.6 & 53.9 & 42.7 \\
\hline Trabajan tiempo completo & 39.5 & 44.3 & 50.6 & 52.7 & 60.0 & 48.9 \\
\hline Se financian con salario $(e n \%)$ & 51.1 & 45.2 & 42.6 & 35.2 & 34.1 & 41.6 \\
\hline Trabajan tiempo parcial & 36.3 & 30.5 & 27.7 & 22.6 & 22.0 & 27.5 \\
\hline Trabajan tiempo completo & 68.4 & 64.6 & 63.4 & 55.5 & 54.7 & 61.7 \\
\hline
\end{tabular}

Para los dos grupos que trabajan con distinta intensidad se observa que las horas trabajadas decrecen levemente por quintiles de rendimiento. Particularmente, la tabla también refleja un aumento marcado de la participación de alumnos cuyo trabajo se vincula con los temas de estudio por quintiles de rendimiento. Estos resultados sugieren que cuando se analiza la relación entre las horas trabajadas y el rendimiento es importante considerar que existen diferencias en los rendimientos de los alumnos según las horas que trabaje el alumno y el grado de vinculación con la carrera del trabajo que realice. Por último, como un indicador de las características económicas por grupos de dedicación laboral, se observa que casi el $62 \%$ de los alumnos que trabajan tiempo completo financian sus estudios sólo a través de su salario, mientras que en el caso de los que trabajan tiempo parcial, el 27.5\% utiliza su salario como fuente. Por quintiles, puede observarse una 
relación decreciente entre el rendimiento y el porcentaje de alumnos que se financian sólo con su salario.

En resumen, se observan patrones contrapuestos según se analicen estas características por condición laboral o por quintiles de rendimiento. Por un lado, clasificando a los alumnos por horas de trabajo, se encuentra que a mayor cantidad de horas trabajadas es más probable que el alumno tenga mayor edad, que sea varón, que esté casado, que lleve más tiempo en la facultad, que su rendimiento sea menor, que estudie menos horas por semana, que su trabajo esté vinculado con la carrera y que se financie sólo su salario. Mientras que si se clasifica a los alumnos por quintiles de rendimiento, se encuentra que los alumnos de mayor rendimiento tienen las características opuestas a las del alumno que más horas trabaja. Este conjunto de resultados es importante para entender que en los datos existen diferencias en el rendimiento según la cantidad de horas trabajadas y las características socio-económicas de los alumnos.

Las disparidades en las características del grupo que trabaja son tales que hasta se podría decir que existen dos tipologías de alumnos que trabajan de acuerdo a cuál sea su dedicación principal. Siguiendo a una clasificación que hace la NCES para Estados Unidos, las evidencias permiten distinguir un grupo de "estudiantes que trabajan" y otro grupo de "trabajadores que estudian" ${ }^{17} \mathrm{La}$ diferencia entre ambos es que, mientras los primeros dedican la mayor parte de su tiempo al trabajo y secundariamente estudian, el segundo grupo principalmente estudia.

\section{III.4 Análisis de las caracteństicas de los alumnos que trabajan por deciles de horas trabajadas}

D ado que en el análisis de la relación trabajo-rendimiento será necesario considerar entonces los efectos diferenciales de las horas trabajadas sobre el rendimiento según la cantidad de horas trabajadas, en esta sección se presenta una evaluación de las características sociales, económicas y académicas de los alumnos que trabajan por deciles de horas trabajadas.

La tabla 3.3 evidencia claramente diferencias observables entre alumnos que trabajan distinta cantidad de horas. Por ejemplo, la edad promedio, la participación de varones, de casados, de alumnos con mayor permanencia y de reingresantes aumentan en los grupos con más horas trabajadas por semana.

Los resultados en términos del rendimiento promedio resultan particularmente interesantes. $\mathrm{Si}$ bien las diferencias observables son leves, nuevamente se observa que el rendimiento alcanza su valor máximo para los alumnos que trabajan pocas horas (aproximadamente 12 horas en promedio de acuerdo a la composición de los deciles). A partir del segundo decil de horas trabajadas el rendimiento decrece a más horas de trabajo.

Vale la pena notar que las horas de estudio también se maximizan en este nivel de horas trabajadas, lo cual refuerza el argumento sobre la relación entre el trabajo, la disciplina del estudiante y su rendimiento. Sin embargo, obsérvese que esto se da sólo para un número moderado de horas trabajadas por semana. Para el resto de los deciles se observa una asociación negativa entre los deciles de horas trabajadas y las horas de estudio, lo cual responde a la idea de que a más horas de trabajo, comienza a operar la restricción temporal del alumno que trabaja, quien termina ajustando la cantidad de horas de estudio favorables a su rendimiento.

${ }^{17}$ National Center of Education and Statistics (NCES), US D epartment of Education, 2002. 
Tabla 3.3 Características de los alumnos que trabajan por deciles de horas trabajadas

\begin{tabular}{|c|c|c|c|c|c|c|c|c|c|}
\hline $\begin{array}{l}\text { Deciles de horas } \\
\text { trabajadas }\end{array}$ & Edad & $\begin{array}{l}\text { Varones } \\
\text { (en \%) }\end{array}$ & $\begin{array}{c}\text { Casados } \\
(\mathrm{en} \%)\end{array}$ & $\begin{array}{c}\text { Se financian } \\
\text { con su salario } \\
\text { (en \%) }\end{array}$ & $\begin{array}{l}\text { Años desde } \\
\text { ingreso }\end{array}$ & $\begin{array}{l}\text { Reingresantes } \\
\text { (en \%) }\end{array}$ & Rendimiento & $\begin{array}{l}\text { Horas de } \\
\text { estudio } \\
\text { semanales }\end{array}$ & $\begin{array}{c}\text { Horas } \\
\text { trabajadas } \\
\text { semanales }\end{array}$ \\
\hline 1 & 23.48 & 41.65 & 10.47 & 18.23 & 4.14 & 4.05 & 2.75 & 26.09 & 5.81 \\
\hline 3 & 24.12 & 44.63 & 12.63 & 19.72 & 4.29 & 4.11 & 2.75 & 28.64 & 19.09 \\
\hline 4 & 24.24 & 45.48 & 13.35 & 24.63 & 4.35 & 4.35 & 2.73 & 27.80 & 23.63 \\
\hline 5 & 25.53 & 48.52 & 18.10 & 39.85 & 4.79 & 6.02 & 2.73 & 25.25 & 29.46 \\
\hline 7 & 26.93 & 56.17 & 23.25 & 55.83 & 5.06 & 6.02 & 2.59 & 22.60 & 39.19 \\
\hline 8 & 26.76 & 58.26 & 23.26 & 58.53 & 5.11 & 6.07 & 2.73 & 22.10 & 41.77 \\
\hline 9 & 26.87 & 63.51 & 24.67 & 62.82 & 5.26 & 6.59 & 2.61 & 21.30 & 46.44 \\
\hline 10 & 27.95 & 70.91 & 31.24 & 70.88 & 5.85 & 7.27 & 2.49 & 20.02 & 55.07 \\
\hline Total & 25.62 & 52.66 & 19.06 & 41.62 & 4.81 & 5.46 & 2.68 & 24.82 & 30.64 \\
\hline
\end{tabular}

\section{III.4.1 Características de los alumnos que trabajan por deciles de horas trabajadas según la vinculación de su trabajo con la carrera}

O tra dimensión en la que se distinguen los alumnos que trabajan entre sí tiene que ver con la vinculación de su trabajo con la carrera que estudian. Por último, se replican algunas columnas del cuadro anterior distinguiendo ahora al grupo que trabaja entre los alumnos que trabajan en temas vinculados con la carrera y los alumnos que trabajan en temas no vinculados con la carrera.

Tabla 3.4 Características de los estudiantes según la vinculación del trabajo con su carrera por deciles de horas trabajadas

\begin{tabular}{c|c|c|c|c|c|c|c|c|c|c|c|c|c}
\hline \multirow{2}{*}{$\begin{array}{c}\text { Deciles de } \\
\text { horas trabajadas }\end{array}$} & \multicolumn{2}{|c|}{ Edad } & \multicolumn{2}{c|}{ Varones (en\%) } & \multicolumn{2}{c|}{ Casados (en\%) } & \multicolumn{2}{c|}{ Años desde ingreso } & \multicolumn{2}{c|}{ Rendimiento } & \multicolumn{2}{c}{ Horas de estudio } \\
\cline { 2 - 11 } & Vinculado & No vinculado & Vinculado & No vinculado & Vinculado & No vinculado & Vinculado & No vinculado & Vinculado & No vinculado & Vinculado & No vinculado \\
\hline 1 & 24.45 & 22.90 & 44.21 & 40.09 & 13.77 & 8.45 & 4.85 & 3.71 & 3.09 & 2.55 & 26.17 & 26.04 \\
2 & 24.90 & 23.18 & 49.05 & 45.12 & 15.54 & 9.87 & 5.14 & 3.87 & 3.13 & 2.56 & 29.57 & 29.71 \\
3 & 25.14 & 23.54 & 45.70 & 44.07 & 16.64 & 10.24 & 4.92 & 3.95 & 3.11 & 2.54 & 27.69 & 29.12 \\
4 & 25.20 & 23.63 & 46.09 & 45.10 & 17.06 & 10.89 & 4.96 & 3.93 & 3.08 & 2.58 & 26.99 & 28.31 \\
5 & 26.33 & 24.85 & 48.76 & 48.33 & 21.40 & 15.36 & 5.42 & 4.29 & 3.02 & 2.52 & 24.13 & 26.31 \\
6 & 27.32 & 25.81 & 51.10 & 49.98 & 24.40 & 19.11 & 5.36 & 4.48 & 3.03 & 2.53 & 23.25 & 24.68 \\
7 & 27.68 & 26.12 & 58.15 & 54.88 & 27.05 & 19.05 & 5.52 & 4.64 & 2.94 & 2.46 & 22.21 & 23.23 \\
8 & 27.48 & 26.28 & 60.95 & 54.97 & 27.76 & 20.31 & 5.57 & 4.67 & 2.98 & 2.47 & 21.38 \\
9 & 27.44 & 26.31 & 67.31 & 60.02 & 28.44 & 20.91 & 5.85 & 4.75 & 2.90 & 2.39 & 20.79 \\
10 & 28.78 & 27.13 & 74.65 & 67.62 & 36.49 & 26.17 & 6.50 & 5.24 & 2.77 & 2.31 & 18.85 & 21.15 \\
\hline
\end{tabular}

Elaboración propia en base al Censo de estudiantes de universidades nacionales de 1994. (SPU).

Como se observa en la tabla 3.4, los alumnos que trabajan también presentan características distintas según su trabajo esté vinculado o no a los contenidos de la carrera que cursan. El resultado más importante aquí es el del rendimiento académico, los alumnos que trabajan en algo vinculado tienen mayores niveles de rendimiento, cualquiera sea el nivel de horas trabajadas.

En la próxima sección se analizará la relación trabajo-rendimiento, dado el conjunto de características que difieren entre los alumnos. Para ello se plantearán distintas especificaciones.

\section{Estimaciones econométricas}

La evidencia preliminar en los datos utilizados refleja que trabajar más horas se relaciona negativamente con el rendimiento académico.

En esta sección se presenta una secuencia de estimaciones realizadas por el método de Mínimos cuadrados ordinarios para analizar empíricamente las preguntas planteadas acerca de la relación trabajo-rendimiento del estudiante. Para ello se estiman modelos del rendimiento educativo 
con distintas especificaciones según las variables utilizadas con relación al trabajo del alumno. En primer lugar (Subsección IV.1), se presentan modelos de regresión para el total de los alumnos censados donde la variable que se utiliza acerca del trabajo del estudiante sólo indica si el alumno trabaja o no. Posteriormente, en el punto IV.2 se estiman modelos exclusivamente para el grupo que trabaja. Allí, en un conjunto de estimaciones se analiza una especificación lineal de la relación entre las horas trabajadas y el rendimiento. Mientras que en otro grupo de modelos, se plantea una relación no lineal entre las horas trabajadas y el rendimiento, a través de la incorporación del cuadrado de las horas trabajadas. En todos los casos se estudia también cómo se altera la relación cuando el trabajo del estudiante está vinculado con la carrera de estudio.

\section{Consideraciones sobre la muestra utilizada}

La fuente de datos que se emplea para realizar el análisis empírico es útil para los propósitos de este estudio por varios motivos. En primer lugar, a partir del Censo de estudiantes de universidades nacionales es posible obtener una medida de rendimiento académico del alumno dada por la cantidad de materias aprobadas por año. O tra ventaja del censo es que contiene información sobre si el alumno trabaja o no, sobre cuántas horas semanales trabaja en el primer caso, sobre la vinculación del trabajo con la carrera y otra referente a la categoría ocupacional. Estas variables son muy útiles para analizar empíricamente cómo se refleja en el rendimiento de los alumnos el hecho de que trabajen, las horas que trabajen y cómo cambia la relación cuando el trabajo se vincula con los temas de estudio, dado otro conjunto de factores explicativos.

No obstante, vale aclarar que la variable ideal para estimar el efecto del trabajo del estudiante sobre su rendimiento hubiera sido un indicador del tiempo que dedicó el estudiante al trabajo en períodos previos al rendimiento observado. Pero este dato sobre la historia laboral del alumno no es captado en el censo. Sólo se cuenta con reportes de las horas semanales que al momento de la encuesta trabaja el estudiante, mientras que el rendimiento se refiere al número promedio de materias que lleva aprobadas desde su ingreso a la facultad. Esto es, en los datos no se puede discernir desde cuándo trabaja el alumno y si su rendimiento fue contemporáneo o no al período en el que el estudiante comenzó a trabajar. ${ }^{18}$ D e hecho, en la relación entre las horas trabajadas y el rendimiento académico puede haber un problema de determinación simultánea, o endogeneidad. Esta posibilidad es reconocida en el trabajo, por ese motivo es necesario adoptar una distinción entre dos acepciones distintas para las variables explicativas según cómo pueden variar respecto al rendimiento educativo. ${ }^{19}$ Se entenderá como factores causales del rendimiento, a las variables exógenas del desempeño educativo y que pueden incidir causándolo (por ej., la edad, el sexo, la nacionalidad, entre otras). En contraste, se entenderá como factores ex plicativos del rendimiento, a aquellas variables que pueden variar conjuntamente con el rendimiento (por ejemplo, la cantidad de horas de estudio o de horas trabajadas). Si bien es cierto que más horas de estudio deberían producir un aumento del rendimiento, también puede ocurrir que un bajo rendimiento induzca mayores horas de estudio, reflejando la causalidad contraria. Asimismo, si bien el trabajo resta tiempo disponible para el estudio y es esperable que influya negativamente sobre el rendimiento, puede ser que los datos reflejen la dirección causal opuesta, subestimando el efecto negativo del trabajo: los alumnos de mayor rendimiento suelen tener más motivación, lo que probablemente los impulse a destinar tiempo al trabajo. En ese caso, si la motivación no se puede incluir en los modelos, podría atribuirse a diferencias en el trabajo, diferencias que provienen de la motivación del alumno. ${ }^{20}$ Los trabajos

\footnotetext{
18 La temporalidad de los determinantes del rendimiento educativo es una de las críticas que se hacen a los modelos basados en el enfoque de la función de producción, ya que los resultados de la educación responden a un proceso acumulativo. Por ejemplo, en Tyler (2003).

19 Esta distinción es realizada en Di G resia et al. (2004).

${ }^{20}$ Como una forma de controlar por este potencial sesgo en los coeficientes de las variables referidas al trabajo del alumno, la incorporación de variables como las horas de estudio y otras características personales en los modelos estimados intenta aproximar la motivación del estudiante.
} 
empíricos que dan cuenta de la potencial endogeneidad, la controlan utilizando principalmente el método de Variables instrumentales. Pero como se adelantara, en las muestras disponibles no suelen encontrarse variables que puedan utilizarse como instrumentos. De modo que los resultados de regresión para este estudio descriptivo no deben verse como una medición de efectos causales sobre el rendimiento, lo cual requiere una especificación que no puede realizarse con los datos disponibles.

En definitiva, los datos del censo de estudiantes de universidades nacionales presentan información valiosa sobre las características del trabajo de los estudiantes para desarrollar el análisis empírico propuesto. Por otra parte, la utilización del censo permite contar con una considerable cantidad de datos representativa del conjunto de estudiantes de todas las universidades nacionales. De más de 615 mil alumnos censados, son utilizables alrededor de 406 mil observaciones. ${ }^{21}$

\section{IV.1Estimaciones para el conjunto de alumnos censados}

En primer lugar se realizaron estimaciones por MCO sobre el total de alumnos censados en las 31 universidades donde se relevó información. El objetivo en esta primera serie de modelos es analizar la relación entre la variable binaria $(=1$ si trabaja, $=0$ si no trabaja) y el rendimiento académico del estudiante. Los resultados para distintas especificaciones son presentados en la tabla 4.1.

Todos los modelos expuestos de aquí en adelante incluyen controles por un conjunto de características observables de los estudiantes según el enfoque de la función de producción del proceso educativo. De modo que para intentar explicar al rendimiento, medida de producto educativo definida por el número de materias aprobadas por año, se utilizó un conjunto de variables que puede subdividirse en cuatro grupos ${ }^{22}$ :

i) Un grupo de variables relacionadas con las características personales del alumno: su edad, sexo, nacionalidad, estado civil, tipo de escuela secundaria (privada o pública), residencia durante el período de clases (si es otra y no la de la facultad implica un costo de traslado), cambio de residencia por estudios, años desde el ingreso, edad al ingreso, fuente de ingresos (trabajo personal, aporte familiar, beca, y combinaciones de las anteriores). Y, como medida de la dedicación o esfuerzo del estudiante, se incluye también la variable horas de estudio.

ii) O tro grupo de variables del entorno familiar. La educación del padre y la educación de la madre medidas en años.

iii) Asimismo, se incluyen controles por universidades y ramas-disciplinas de estudio. ${ }^{23}$ Estas incorporaciones se fundamentan en que las universidades son un conjunto heterogéneo en cuanto a tamaño, ubicación geográfica, tradición, técnicas de aprendizaje, entre otros factores. ${ }^{24}$ Por lo tanto, estas diferencias del ámbito universitario deben ser consideradas en el modelo. Análogamente, el abanico de ramas-disciplinas de estudio está formado por carreras muy diversas. Es necesario controlar entonces por la autoselección que realizan los estudiantes entre carreras elegidas conociendo su motivación, limitaciones y ventajas asociadas respecto a los temas de estudio y futuro laboral. ${ }^{25} 26$

\footnotetext{
${ }^{21}$ Esta diferencia se debe a que parte de los alumnos no completaron todo el cuestionario del censo.

${ }^{22}$ El conjunto de variables utilizadas se describe en detalle en el Anexo 1.

23 En el Anexo 2 se describe la composición de las ramas de estudio en términos de disciplinas. La variable ramadisciplina es una variable de interacción entre la variable rama y la variable disciplina. Por ejemplo, la carrera de Contador público pertenece a la Rama Ciencias Sociales y al conjunto de disciplinas de Administración, Ciencias Económicas y Organización. (Anuario 99-00 de Estadísticas Universitarias).

24 Ver Ripani y Porto (2003), Gertel (1999) y Anuario 99-00 de Estadísticas Universitarias.

25 Incluir controles por carrera implicaría incluir 899 variables binarias. Por ello, como una aproximación válida, sólo se incluyen 17 controles por agrupamientos de carreras según su rama-disciplina de estudio. Posteriormente se comenta sobre las estimaciones realizadas para uno de los agrupamientos analizados por carreras, en ramas-disciplinas.
} 
iv) Por último, el grupo de variables más relevante para responder las preguntas planteadas en este estudio se refiere a las variables relacionadas con el trabajo del estudiante: En el primer grupo de modelos se utiliza una variable binaria "trabaja", luego se incluye otra variable binaria que indica si el trabajo se vincula o no con la carrera de estudio, mientras que en los modelos presentados en la Subsección IV.2, estimados sólo para grupos de alumnos que trabajan, se utilizan las horas trabajadas y la dummy de trabajo vinculado.

En síntesis, el modelo planteado podría expresarse como:

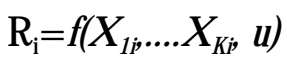

D onde: Ri es una medida de rendimiento académico del estudiante $\mathrm{i}$, en este caso el número de materias aprobadas por año; $X_{1 i}, \ldots, X_{K i}$ constituyen el conjunto de $\mathrm{K}$ variables explicativas que pueden incidir en el rendimiento académico del estudiante $\mathrm{i}$; y u representa a los factores no observables,

D e modo de concentrar el análisis en la relación trabajo-rendimiento, los resultados para las variables no referidas al trabajo del estudiante se comentarán sucintamene sólo en este primer conjunto de estimaciones.

En la tabla 4.1 se exponen los resultados para el total censado (que incluye a los alumnos que trabajan y los que no trabajan). Entre el grupo de variables personales, se encuentra que los alumnos de mayor edad tienen un mayor rendimiento esperado. Este resultado puede estar asociado a que en la muestra para el total del censo hay una sobrerepresentación de alumnos jóvenes, entre los cuales es mayor la propensión a abandonar la carrera en poco tiempo desde el ingreso ${ }^{27}$ Otro resultado es que el alumno varón tiene menor rendimiento comparado con el de la mujer, lo cual suele explicarse por la mayor motivación de las mujeres para el estudio u otras tareas académicas.

En cuanto a la nacionalidad, se encuentra que los estudiantes extranjeros tienen un menor rendimiento que los argentinos, adjudicable a problemas de adaptación. El estado civil es también un factor significativo y, en particular, ser soltero se asocia positivamente con el rendimiento. Por otra parte, referente a la historia académica del estudiante, se encuentra que los que provienen de una educación secundaria privada tienen un mayor rendimiento que los alumnos que pertenecieron a una escuela secundaria pública.

Los alumnos que deben trasladarse desde otra jurisdicción hacia la facultad tienen un rendimiento menor respecto a los que viven en la misma localidad, resultado que puede adjudicarse al tiempo y esfuerzo de transporte. Otro resultado indica que los alumnos que llevan más tiempo en la facultad rinden menor cantidad de materias por año, atribuible a que con los años el estudiante va tomando nuevas obligaciones como el trabajo, el matrimonio, entre otros cambios.

El cambio de residencia durante el período de estudios se observa como un factor positivo y significativo para el rendimiento: esto sugiere que el costo de oportunidad que implica haber cambiado la residencia tiene efectos que superan al del desarraigo.

Los resultados para el coeficiente de la edad al ingreso del estudiante señalan que cuanto menor es la edad con la que se ingresa a la facultad, mayor el rendimiento esperado del alumno.

En cuanto al número de horas de estudio semanales, como se esperaba, los alumnos que estudian más horas por semana, incluyendo el tiempo de cursadas, rinden un mayor número de materias por año.

En otro orden, con respecto al efecto del entorno familiar del estudiante, los resultados indican que a mayor educación de los padres, mayor es el rendimiento esperado de los hijos.

26 También se incluyen variables referidas a la calidad de la universidad en términos de gasto por alumno promedio, composición promedio del cuerpo docente y la cantidad de docentes equivalentes en la universidad, detalladas en el Anexo 1.

${ }^{27} \mathrm{Al}$ momento del censo, los mejores alumnos de la cohorte más antigua que se graduaron a tiempo no pudieron ser incluidos. 
La fuente de financiamiento de los estudios es una variable estadísticamente significativa. Se encuentra que comparado con el financiamiento a partir del trabajo personal, el resto de las fuentes incide positivamente, y es mayor el rendimiento esperado para los estudiantes que se financian mediante la combinación de beca y aporte familiar.

\section{Análisis de la relación "trabaja"-rendimiento}

El resultado más relevante para el trabajo en esta primera aproximación presentada en la tabla 4.1 surge de observar el coeficiente de la relación entre la variable "trabaja" y la cantidad de materias aprobadas por año. En el primer modelo de regresión sólo se incluyó este factor referido al trabajo del estudiante. En este sentido, es posible comparar el rendimiento esperado para un alumno que reporta que trabaja con respecto al que no, dadas el resto de sus características.

Como se observa para todas las especificaciones de la tabla, en una regresión para el total de alumnos censados, la variable binaria "trabaja" resulta significativa. En contraste con lo esperado, pero en relación con lo señalado en la sección III, el signo refleja que el estudiante que trabaja tiene un mayor rendimiento respecto al que no trabaja.

En esta estimación para el total de la muestra, el signo positivo asociado a la variable binaria "trabaja" es plausible de distintas interpretaciones.

Podría adjudicarse a que hay alumnos cuyas actividades laborales son complementarias de sus carreras. Este argumento se sostiene en la teoría de learning by doing o aprender haciendo, que señala que la transferencia de conocimiento es más efectiva a través de la práctica de lo que se estudia. La idea de learning by doing ha sido sostenida por filósofos y psicólogos y, según Stern et al. (1997), sobre esta misma teoría se han diseñado políticas educativas para países en desarrollo como por ejemplo el Acta de Oportunidades de escuela-trabajo de 1994 en Estados Unidos, basadas en el reconocimiento del aprendizaje basado en el trabajo.

También el signo positivo puede deberse, como suele argumentarse, a que además de facilitar la transmisión de conocimientos académicos, el trabajo enseña y refuerza actitudes como la responsabilidad y madurez que favorecen los resultados académicos. Asimismo, Lillydahl (1990) señala que el trabajo puede llegar a favorecer conductas en los estudiantes beneficiosas para sus logros académicos.

Sin embargo, el signo positivo encontrado también indica que puede haber un problema de endogeneidad al estimar la relación entre el trabajo y el rendimiento por Mínimos cuadrados ordinarios (MCO). Como señalan Stinebrickner y Stinebrickner (2003), los métodos de regresión simple podrían subestimar el efecto negativo del trabajo sobre el rendimiento. En tal caso, el coeficiente de la variable "trabaja" podría estar captando aspectos no observados del estudiante, como la motivación o la inteligencia que pueden estar correlacionados con la decisión de trabajar del alumno. En consecuencia, este primer resultado debe verse con cautela ya que la heterogeneidad no observada asociada a la decisión de trabajar de los estudiantes puede llevar a estimaciones sesgadas del efecto del trabajo en el rendimiento de los estudiantes. ${ }^{28}$

Asimismo, la variable binaria "trabaja" puede ser una medida imperfecta para captar la relación entre el trabajo del estudiante y su rendimiento, ya que no considera diferencias en la intensidad del trabajo ni en el tipo de trabajo. Esto es, esta regresión no controla por la cantidad de horas que trabaja el individuo, ni por el tipo de ocupación. Por otra parte, en la sección III se pudo observar que existen diferencias en el rendimiento y otras características de los estudiantes de acuerdo al número de horas que trabajen. En la siguiente subsección se presenta la estimación de un modelo sólo para el grupo de alumnos que trabaja, donde el trabajo del estudiante será incluido en términos de horas trabajadas.

${ }^{28}$ En la siguiente sección se comentan los resultados de la estimación por Mínimos cuadrados en dos etapas como un método alternativo a MCO y Variables instrumentales 
Tabla 4.1 Estimaciones por MCO para el total de alumnos censados

\begin{tabular}{|c|c|c|c|c|c|c|c|c|}
\hline \multirow{2}{*}{ Variables explicativas } & \multicolumn{2}{|c|}{ a) } & \multicolumn{2}{|c|}{ b) } & \multicolumn{2}{|c|}{ c) } & \multicolumn{2}{|c|}{ d) } \\
\hline & Coef. & p-value & Coef. & $\mathrm{p}$-value & Coef. & $\mathrm{p}$-value & Coef. & p-value \\
\hline Edad & 0.305 & 0.000 & 0.310 & 0.000 & 0.341 & 0.000 & 0.346 & 0.000 \\
\hline Sexo (1:varón, 0: mujer) & -0.046 & 0.000 & -0.028 & 0.000 & -0.058 & 0.000 & -0.041 & 0.000 \\
\hline Nacionalidad (1:extranjero, 0:argentino) & -0.052 & 0.000 & -0.051 & 0.000 & -0.046 & 0.001 & -0.045 & 0.002 \\
\hline Estado civil: unido & -0.019 & 0.267 & 0.016 & 0.361 & -0.012 & 0.529 & 0.024 & 0.192 \\
\hline Estado civil: casado & 0.126 & 0.000 & 0.154 & 0.000 & 0.129 & 0.000 & 0.157 & 0.000 \\
\hline Estado civil: separado & -0.075 & 0.010 & -0.044 & 0.130 & -0.041 & 0.185 & -0.009 & 0.765 \\
\hline Estado civil:divorciado & -0.172 & 0.000 & -0.138 & 0.000 & -0.171 & 0.000 & -0.137 & 0.000 \\
\hline Estado civil:viudo & 0.708 & 0.000 & 0.689 & 0.000 & 0.702 & 0.000 & 0.680 & 0.000 \\
\hline Estado civil:soltero (base) & & & & & & & & \\
\hline Escuela Secundaria privada (1:priv, 0:pública) & 0.106 & 0.000 & 0.101 & 0.000 & 0.100 & 0.000 & 0.095 & 0.000 \\
\hline Resid. durante clases (1: otra, 0: misma) & -0.034 & 0.000 & -0.034 & 0.000 & -0.036 & 0.000 & -0.036 & 0.000 \\
\hline Cambio resid. debido a estudios (1:si, 0: no) & 0.122 & 0.000 & 0.118 & 0.000 & 0.125 & 0.000 & 0.122 & 0.000 \\
\hline Años desde el ingreso & -0.357 & 0.000 & -0.356 & 0.000 & -0.405 & 0.000 & -0.405 & 0.000 \\
\hline Edad al ingreso & -0.296 & 0.000 & -0.297 & 0.000 & -0.331 & 0.000 & -0.332 & 0.000 \\
\hline Horas semanales de estudio (con clases) & 0.012 & 0.000 & 0.011 & 0.000 & 0.012 & 0.000 & 0.011 & 0.000 \\
\hline Años de educación del padre & 0.018 & 0.000 & 0.017 & 0.000 & 0.016 & 0.000 & 0.016 & 0.000 \\
\hline Años de educación de la madre & 0.018 & 0.000 & 0.017 & 0.000 & 0.017 & 0.000 & 0.016 & 0.000 \\
\hline Fuente de ing.: trabajo personal (base) & & & & 0.000 & & & & \\
\hline Fuente de ing.: aporte familiar & & & 0.282 & 0.000 & & & 0.269 & 0.000 \\
\hline Fuente de ing.: trabajo personal y ap. Familiar & & & 0.195 & 0.000 & & & 0.195 & 0.000 \\
\hline Fuente de ing.: Sólo beca de estudios & & & 0.470 & 0.000 & & & 0.471 & 0.000 \\
\hline Fuente de ing.: beca de estudios y ap. Familiar & & & 0.602 & & & & 0.561 & 0.000 \\
\hline Fuente de ing.: otras & & & 0.246 & & & & 0.241 & 0.000 \\
\hline Trabaja (1:si, 0:no) & 0.055 & 0.000 & 0.173 & 0.000 & -0.117 & 0.000 & -0.007 & 0.423 \\
\hline Trabajo vinculado con la carrera (1:si, 0:no) & & & & & 0.457 & 0.000 & 0.456 & 0.000 \\
\hline Constante & -5.298 & 1.000 & 2.668 & 0.998 & 3.023 & 0.998 & 2.612 & 0.000 \\
\hline "No de obs. & $\overline{406494}$ & & 405065 & & $\overline{404082}$ & & $\overline{402722}$ & \\
\hline$F(.,)$. & 1483.0 & & 1397.1 & & 1630.0 & & 1553.1 & \\
\hline Prob $>F$ & 0.000 & & 0.000 & & 0.000 & & 0.000 & \\
\hline R-squared & 0.189 & & 0.192 & & 0.208 & & 0.210 & \\
\hline Adj.R-Squared & 0.189 & & 0.192 & & 0.208 & & 0.210 & \\
\hline
\end{tabular}

Elaboración propia en base al Censo de estudiantes de universidades nacionales de 1994. (SPU).

Nota: Las especificaciones incluyen también controles por universidades (31 dummies) y por rama-disciplina (17 dummies).

También se controla por variables indicadoras de calidad específicas de la universidad como gasto promedio por alumno,

composición promedio del cuerpo docente y cantidad de docentes equivalentes.

Pero previamente, se describirán los resultados de incluir una variable de control que indica si el trabajo del estudiante está vinculado o no con la carrera que estudia. En la tercera columna de la tabla se presenta el modelo anterior incluyendo la variable binaria "trabajo vinculado con la carrera" (1: si se vincula, 0: si no), un indicador de la complementariedad de trabajo que realiza el estudiante con la carrera que cursa. El coeficiente indica que como se esperaba, aquel alumno que trabaja en tareas complementarias a sus estudios tiene un rendimiento superior respecto a aquel cuyo trabajo no está asociado a la carrera. Este resultado es consistente con estudios previos, por ejemplo Stern et al. (1997), encuentran que el efecto negativo de las horas trabajadas es menor cuando se trata de trabajos vinculados a los temas de estudio. En ese sentido, por ejemplo, Pauly et al. (1995) sugieren que una estrategia para reducir o eliminar los efectos negativos del trabajo de los estudiantes puede ser la creación de vínculos más explícitos entre los temas de estudio y los trabajos. Además, si se observa el coeficiente de la variable "trabaja", puede encontrarse que ha cambiado de signo. Esto comprueba que la medida anterior puede estar captando otros efectos, en este caso, hay indicios de que reflejaba el efecto complementario del trabajo vinculado.

La hipótesis de las ventajas del trabajo vinculado respecto al no vinculado en términos de rendimiento, ceteris paribus otras condiciones del trabajo, será nuevamente introducida en el análisis subsiguiente. También se analizarán modelos por grupos de alumnos de acuerdo a la dicotomía trabajo vinculado-trabajo no vinculado. 
En lo que sigue se analiza la pregunta de cómo se relacionan las horas que trabaja el estudiante con su rendimiento académico a través de un conjunto de estimaciones considerando solamente al grupo de alumnos que trabaja.

\section{IV.2 Estimaciones para el grupo de alumnos que trabaja}

\section{IV.2.1 Análisis de la especificación lineal de la relación horas trabajadas- rendimiento}

Uno de los objetivos principales del trabajo es analizar cómo se relacionan las horas que trabaja el estudiante con su nivel de rendimiento académico. Una posibilidad es plantear un modelo en el que la relación sea lineal. En otros términos, que la productividad marginal de las horas trabajadas en promedio sea la misma para cualquier nivel de horas trabajadas. Para analizar esto se realizaron estimaciones por Mínimos cuadrados ordinarios para el grupo de alumnos que trabajan incorporando en el modelo a las horas trabajadas semanales. La muestra se reduce a casi 226 mil alumnos que reportaron las horas trabajadas.

Los resultados de la estimación para el grupo de alumnos que trabaja se presentan en la tabla 4.2. En la especificación lineal se encuentra que el coeficiente de las horas semanales trabajadas por los alumnos es negativo y estadísticamente significativo. Esto sugiere que los alumnos que más horas trabajan por semana rinden una menor cantidad de materias por año.

Tabla 4.2 Estimaciones por MCO para el grupo que trabaja y según vinculación del trabajo con la carrera.

Especificación lineal

\begin{tabular}{|c|c|c|c|c|c|c|c|c|}
\hline \multicolumn{5}{|c|}{$\begin{array}{lc}\text { Variable dependiente: } \text { Cantidad de materias aprobadas por año (rendimiento académico } \\
\text { Grupo de alumnos: } & \text { Total que trabaja }\end{array}$} & \multirow{2}{*}{\multicolumn{2}{|c|}{$\frac{\text { Trabajo vinculado }}{\text { C) }}$}} & \multirow{2}{*}{\multicolumn{2}{|c|}{$\frac{\text { Trabajo no vinculado }}{\text { a) }}$}} \\
\hline & \multicolumn{2}{|c|}{ a) } & \multicolumn{2}{|c|}{ D) } & & & & \\
\hline Variables explicativas & Coef. & $\overline{p \text {-value }}$ & Coef. & p-value & Coef. & $p$-value & Coef. & p-value \\
\hline Edad & 0.305 & 0.000 & 0.299 & 0.000 & 0.320 & 0.000 & 0.277 & 0.000 \\
\hline Sexo (1:varón, 0: mujer) & -0.048 & 0.000 & -0.053 & 0.000 & -0.080 & 0.000 & -0.031 & 0.001 \\
\hline Nacionalidad (1:extranjero, 0:argentino) & -0.079 & 0.000 & -0.073 & 0.000 & -0.083 & 0.003 & -0.061 & 0.007 \\
\hline Estado civil: unido & 0.054 & 0.010 & 0.053 & 0.012 & 0.010 & 0.756 & 0.077 & 0.004 \\
\hline Estado civil: casado & 0.192 & 0.000 & 0.173 & 0.000 & 0.165 & 0.000 & 0.198 & 0.000 \\
\hline Estado civil: separado & 0.022 & 0.535 & 0.041 & 0.238 & -0.028 & 0.610 & 0.095 & 0.030 \\
\hline Estado civil:divorciado & -0.100 & 0.004 & -0.097 & 0.005 & -0.213 & 0.000 & 0.023 & 0.622 \\
\hline Estado civil:viudo & -0.100 & 0.176 & -0.100 & 0.177 & -0.041 & 0.718 & -0.107 & 0.270 \\
\hline Estado civil:soltero (base) & & & & & & & & \\
\hline Escuela Secundaria privada (1:priv, 0:pública) & 0.101 & 0.000 & 0.090 & 0.000 & 0.089 & 0.000 & 0.091 & 0.000 \\
\hline Resid. durante clases (1: otra, 0: misma) & -0.038 & 0.000 & -0.043 & 0.000 & -0.054 & 0.000 & -0.027 & 0.010 \\
\hline Cambio resid. debido a estudios (1:si, 0: no) & 0.142 & 0.000 & 0.144 & 0.000 & 0.236 & 0.000 & 0.079 & 0.000 \\
\hline Años desde el ingreso & -0.372 & 0.000 & -0.377 & 0.000 & -0.427 & 0.000 & -0.331 & 0.000 \\
\hline Edad al ingreso & -0.293 & 0.000 & -0.290 & 0.000 & -0.315 & 0.000 & -0.266 & 0.000 \\
\hline Horas de estudio semanales (con clases) & 0.010 & 0.000 & 0.010 & 0.000 & 0.008 & 0.000 & 0.012 & 0.000 \\
\hline Años de educación del padre & 0.017 & 0.000 & 0.014 & 0.000 & 0.016 & 0.000 & 0.013 & 0.000 \\
\hline Años de educación de la madre & 0.017 & 0.000 & 0.015 & 0.000 & 0.016 & 0.000 & 0.014 & 0.000 \\
\hline Fuente de ing.: trabajo personal (base) & & & & & & & & \\
\hline Fuente de ing.: aporte familiar & 0.216 & 0.000 & 0.207 & 0.000 & 0.371 & 0.000 & 0.075 & 0.000 \\
\hline Fuente de ing.: trabajo $\mathrm{p}$ & 0.144 & 0.000 & 0.142 & 0.000 & 0.186 & 0.000 & 0.100 & 0.000 \\
\hline Fuente de ing.: Sólo beca de estudios & 0.427 & 0.000 & 0.413 & 0.000 & 0.468 & 0.000 & 0.352 & 0.001 \\
\hline Fuente de ing.: beca de estudios y ap. Familiar & 0.664 & 0.000 & 0.593 & 0.000 & 0.694 & 0.000 & 0.451 & 0.000 \\
\hline Fuente de ing.: otras & 0.195 & 0.000 & 0.186 & 0.000 & 0.432 & 0.000 & 0.011 & 0.811 \\
\hline Horas trabajadas semanales & -0.003 & 0.000 & -0.004 & 0.000 & -0.002 & 0.000 & -0.005 & 0.000 \\
\hline Trabajo vinculado con la carrera (1:si, 0:no) & & & 0.458 & 0.000 & & & & \\
\hline Constante & 1.585 & 1.000 & 1.015 & 0.152 & 2.241 & 1.000 & 1.096 & 1.000 \\
\hline № de obs. & 225830 & & 223598 & & 101007 & & 122591 & \\
\hline $\mathrm{F}(. .)$. & 901.5 & & 981.5 & & 468.3 & & 468.0 & \\
\hline Prob $>F$ & 0.000 & & 0.000 & & 0.000 & & 0.000 & \\
\hline R-squared & 0.218 & & 0.233 & & 0.243 & & 0.211 & \\
\hline Adj.R-Squared & 0.218 & & 0.232 & & 0.242 & & 0.211 & \\
\hline
\end{tabular}

Elaboración propia en base al Censo de estudiantes de universidades nacionales de 1994. (SPU).

Nota: Las especificaciones incluyen también controles por universidades (31 dummies) y por rama-disciplina (17 dummies). 
Si bien el efecto observado es pequeño, como era de esperar dado que las horas trabajadas están definidas por semana y el rendimiento en términos anuales, trabajar una hora menos por semana representaría 0.16 materias aprobadas menos por año. Este resultado es consistente con trabajos como el de Tyler (2003), que encuentra una relación negativa por el método de MCO entre las horas trabajadas y la medida de rendimiento académico utilizada. En cuanto a la magnitud del resultado, señala que si bien la relación es negativa tanto por el método de MCO como el de Variables instrumentales, el efecto es más fuerte en la estimación por éste último método. Esta diferencia la atribuye a que la estimación por MCO tiende a subestimar el efecto negativo del trabajo sobre el rendimiento si, condicional en las características observables, los alumnos de más alto rendimiento tienden a trabajar más. Por ello, si bien se reconoce que los resultados por MCO pueden estar subestimando el efecto negativo, esta prueba no se ha podido mostrar aquí por la falta de instrumentos significativos en los datos para aplicar Variables instrumentales. ${ }^{29}$ No obstante, a los fines descriptivos de este trabajo, entre los cuales no se busca establecer magnitudes de efectos, los signos encontrados son consistentes con los de estimaciones que controlan por la presencia de endogeneidad. De todos modos se reconoce que, considerando la evidencia de otras pruebas realizadas por MCO en dos etapas y las evidencias de otros estudios por Variables instrumentales, los efectos presentados hubieran sido mayores.

Stinebrickner y Stinebrickner (2003) también encuentran un efecto negativo de las horas trabajadas sobre el rendimiento a través de la estimación por el método de Variables instrumentales, pero para hacerlo, cuentan con una muestra muy única que contiene un instrumento dado por las horas semanales de trabajo que se les asigna a los estudiantes en el primer semestre de ingreso a la carrera, por lo que esas horas sólo están correlacionadas con el rendimiento a través de su efecto sobre el mismo y no al revés.

La explicación a esta relación negativa entre las horas trabajadas y el rendimiento académico se basa en la restricción temporal del estudiante. Dada una dotación de tiempo, como suele argumentarse, las horas dedicadas a trabajar restan tiempo disponible para desarrollar actividades académicas como el estudio, que se observan beneficiosas para el rendimiento. En consecuencia, si las horas de estudio fueran sustituidas por tiempo de trabajo, el trabajo incidiría negativamente en el rendimiento educativo. ${ }^{30}$

Analizando esta interpretación, se estimó un modelo similar al anterior con las horas de estudio como variable dependiente, de modo de investigar cómo inciden las horas trabajadas en las horas de estudio. Se encuentra que el coeficiente de las horas trabajadas es negativo y significativo en todas las especificaciones estimadas. ${ }^{31}$

\section{Horas trabajadas y vinculación del trabajo con la camera}

O tro objetivo de este trabajo es analizar qué ocurre con el efecto de las horas trabajadas cuando el trabajo que realiza el estudiante está vinculado con la carrera que cursa. En primer lugar, se estimó el mismo modelo presentado anteriormente pero incluyendo un control a través de una variable binaria que indica si el trabajo está relacionado con la carrera que cursa (=1 si el trabajo está vinculado, $=0$ si no). Estos resultados se exponen en la segunda columna de la tabla 4.2. Luego en la tercera y cuarta columna se presentan los resultados de la estimación para el grupo de estudiantes que trabaja en algo vinculado en comparación con los resultados para el grupo que no trabaja en algo vinculado.

${ }^{29}$ Como alternativa a la utilización del método de Variables instrumentales, todas las estimaciones presentadas en esta sección fueron estimadas también por el método de Mínimos cuadrados en dos etapas. Se obtienen los mismos signos e incluso los efectos encontrados son más fuertes a partir de este último método.

${ }^{30}$ Recapitulando, en la subsección III.3 se observó también una asociación negativa entre los deciles de horas trabajadas y las horas de estudio.

${ }^{31}$ Estos y otros resultados que se comentan, pueden ser provistos. 
En el modelo para el total de alumnos que trabaja que incluye la variable binaria "trabajo vinculado con la carrera", se encuentra que se mantiene el coeficiente negativo y significativo de las horas trabajadas y que el alumno que trabaja en algo vinculado tiene un rendimiento mayor respecto a aquel alumno que trabaja en temas no vinculados con el estudio. En términos cuantitativos, un alumno que trabaja en algo vinculado aprueba en promedio 0.45 materias más por año en comparación con un alumno cuyo trabajo no tiene relación con los temas que estudia.

Este resultado, como se señaló anteriormente, es consistente con el argumento de la teoría de learning by doing y con los resultados de trabajos empíricos que analizan el impacto diferencial de las horas trabajadas en trabajos vinculados con la carrera respecto al de las horas trabajadas en trabajos no vinculados. El grado de vinculación del trabajo con el plan de estudios es un factor importante en el análisis de la relación entre el trabajo y el rendimiento académico. De hecho ha sido motivo de análisis de varios artículos como Stone et al. (1990), G reenberger y Steinberg (1986), Stern et al. (1997), Pauly (1995), entre otros. Por ejemplo, Stone et al. resaltan que la mayor parte de los estudiantes de escuelas secundarias en Estados Unidos trabajan en empleos no relacionados con los temas que estudian. Greenberger y Steinberg señalan que la mayor parte de los estudiantes universitarios y secundarios no trabajan para aplicar o extender lo que aprenden, sino para consumir en el presente o cubrir sus gastos. Mientras que Stern (1997) concluye que los trabajos no vinculados con la carrera generan una brecha entre el aprendizaje en la escuela y el trabajo.

\section{Relación Horas de trabajo vinculado-rendimiento}

En las dos últimas columnas de la tabla 4.2 puede observarse cómo se traduce el resultado anterior en términos de horas trabajadas en un trabajo vinculado versus horas trabajadas en un trabajo no vinculado. En comparación con el procedimiento anterior, esta especificación por grupos permite controlar el sesgo de autoselección de los alumnos entre trabajos vinculados y no vinculados y además posibilita analizar más directamente la relación de las horas trabajadas en trabajos vinculados y no vinculados con el rendimiento académico.

En la tercera columna se presenta la estimación realizada sólo para el grupo que trabaja en algo vinculado. Se encuentra que el coeficiente de las horas trabajadas es significativo y negativo. La interpretación es que aún cuando el alumno trabaje en temas vinculados con la carrera, una hora adicional semanal de trabajo incide negativamente sobre la cantidad de materias que aprueba por año, dada su restricción temporal.

\section{Relación Horas de trabajo no vinculado-rendimiento}

Se realizó comparativamente el análisis de la incidencia de las horas trabajadas sobre el rendimiento, exclusivamente para el grupo cuyo trabajo no se vincula con el tema de estudio. Como resultado, en las últimas dos columnas, se encuentra que el coeficiente de las horas trabajadas es aún más negativo que el anterior para el grupo que trabaja en algo vinculado a su carrera. (-0.005 vs.0.002). ${ }^{32}$ Este resultado es consistente con los de otros autores ya comentados como el de Stern et al. (1997), por ejemplo.

En resumen, hasta aquí se encuentran las primeras evidencias para la pregunta de cómo afectan las horas trabajadas del estudiante su desempeño académico. Si se plantea una relación lineal entre las horas trabajadas y el rendimiento del alumno, se observa que una hora adicional semanal en el trabajo del estudiante universitario incide negativamente en su rendimiento. Incluso esta relación

\footnotetext{
${ }^{32}$ Los resultados se mantienen si se agregan controles por categoría ocupacional del alumno. (Esto es, si es patrón, empleado sector público, empleado sector privado, profesional universitario cuenta propista, cuenta propista no profesional, o empleado del servicio doméstico o trabajador familiar).
} 
negativa puede reforzarse si el trabajo que realiza el alumno no está relacionado con los temas que estudia.

En contraste con los resultados de la subsección anterior, donde se encontraba que el alumno que trabaja tiene un mayor rendimiento respecto al que no trabaja, se observa que las horas trabajadas se asocian negativamente con el rendimiento del alumno. Esta ambigüedad puede tener más de una explicación. Una de ellas es que la variable binaria "trabaja" es más proclive a reflejar otras características que pueden llegar a ser buenas del trabajo, y de hecho puede estar captando condiciones no observables del estudiante. Por otra parte, la variable "horas trabajadas por semana" parece un indicador más apropiado de la restricción temporal que implica trabajar para el alumno, que es la vía a través de la cual se argumenta que el trabajo puede ser perjudicial para su desempeño académico. Asimismo, incluir sólo la variable "trabaja" no permite distinguir por las diferencias en la intensidad de horas trabajadas ni por la vinculación entre el trabajo y los temas de estudio.

Pero vale la pena preguntarse si el coeficiente para las horas trabajadas es negativo cualquiera sea el nivel de horas trabajadas, ya que seguramente trabajar una hora más semanalmente no tenga la misma incidencia sobre el rendimiento para el alumno que trabaja 10 horas por semana que para el que trabaja 50 horas semanales. En otros términos, sería interesante analizar si se observan distintos efectos dependiendo la intensidad con la que el alumno trabaja.

\section{IV.2.2 Análisis de la especificación no lineal de la relación horas trabajadas- rendimiento}

O tro de los objetivos de este trabajo es entonces analizar si existe una relación no lineal entre las horas trabajadas y el rendimiento del estudiante. Para estudiar esta hipótesis se incluye en el modelo a la variable horas trabajadas y al cuadrado de las horas trabajadas. Con esta inclusión se quiere observar además del signo, la curvatura de la relación entre las horas trabajadas y el rendimiento académico.

Como puede observarse en la primera columna de la tabla 4.3, el efecto de las horas trabajadas es positivo y estadísticamente significativo, mientras que el de su cuadrado es negativo y significativo.

Los resultados indican que una hora adicional de trabajo se asocia positivamente con el rendimiento para una cantidad moderada de horas trabajadas. Pero también que esta productividad marginal del alumno es decreciente. A partir de los resultados se estima que alcanza un máximo de rendimiento académico en 14.5 horas trabajadas semanales y luego se torna negativa. Esto indica una relación no lineal entre las horas trabajadas y el rendimiento del alumno, por cuanto el efecto del tiempo dedicado al trabajo dependerá de la cantidad de horas trabajadas.

En un nivel bajo de horas trabajadas parece cierto entonces que el trabajo del estudiante incida positivamente en su desempeño, por cuanto hasta ese nivel se aprovechan las ventajas del mayor sentido de responsabilidad, o bien de la complementariedad de las tareas curriculares en el ámbito laboral. Sin embargo, la relación positiva es decreciente a mayor cantidad de horas trabajadas y con una carga horaria superior a las 14.5 horas semanales, el tiempo disponible para el estudio probablemente es reemplazado por el tiempo dedicado al trabajo, lo cual incide negativamente sobre el desempeño del alumno.

Estos resultados compatibilizan con los dos argumentos contrapuestos acerca de la relación entre el trabajo del estudiante y su rendimiento. Si bien se observa que el trabajo tiene incidencia positiva, esta se mantiene para un nivel bajo de horas trabajadas. Lillydahl (1990) también encuentra un efecto no lineal. La relación es positiva hasta 13.5 horas semanales trabajadas, utilizando el G PA como medida de rendimiento y a través del método de Mínimos cuadrados en dos etapas. Shill et al (1985) también encuentran una relación curvilínea significativa entre las horas trabajadas y el GPA. El límite de horas en este caso alcanza las 20 horas semanales.

También estos resultados se asocian con la tabla 3.3 por deciles de horas trabajadas, donde se observa que en el segundo decil de horas trabajadas el rendimiento y las horas de estudio alcanzan su valor máximo. 
Tabla 4.3 Estimaciones por MCO para los alumnos que trabajan según la vinculación del trabajo con la carrera.

Especificación no lineal

\begin{tabular}{|c|c|c|c|c|c|c|c|c|}
\hline \multirow{2}{*}{ Grupo de alumnos: } & \multicolumn{4}{|c|}{ Total que trabaja } & \multirow{2}{*}{\multicolumn{2}{|c|}{$\frac{\text { Trabajo vinculado }}{\text { c) }}$}} & \multirow{2}{*}{\multicolumn{2}{|c|}{\begin{tabular}{|c|} 
Trabajo no vinculado \\
d)
\end{tabular}}} \\
\hline & & & & & & & & \\
\hline Variables explicativas & Coef. & p-value & Coef. & p-value & Coef. & p-value & Coef. & p-value \\
\hline Horas trabajadas semanales & 0.0029 & 0.001 & 0.0017 & 0.050 & 0.0049 & 0.000 & -0.0017 & 0.121 \\
\hline Horas trabajadas semanales al cuadrado & -0.0001 & 0.000 & -0.0001 & 0.000 & -0.0001 & 0.000 & -0.0001 & 0.002 \\
\hline Trabajo vinculado con la carrera (1:si, 0:no) & & & 0.4572 & 0.000 & & & & \\
\hline Constante & 1.4895 & 1.000 & 0.9479 & 0.181 & 2.1614 & 1.000 & 1.0623 & 1.000 \\
\hline № de obs. & 225830 & & 223598 & & 101007 & & 122591 & \\
\hline$F(.)$. & 901.5 & & 968.2 & & 462.1 & & 461.5 & \\
\hline Prob $>F$ & 0.000 & & 0.000 & & 0.000 & & 0.000 & \\
\hline R-squared & 0.218 & & 0.233 & & 0.243 & & 0.211 & \\
\hline Adj.R-Squared & 0.218 & & 0.232 & & 0.242 & & 0.211 & \\
\hline
\end{tabular}

Elaboración propia en base al Censo de estudiantes de universidades nacionales de 1994. (SPU).

Nota: El modelo estimado sólo difiere del presentado en la tabla anterior en que se incluyen las horas trabajadas

al cuadrado.

\section{Horas trabajadas y vinculación del trabajo con la carrera}

O tra pregunta que interesa en este estudio es cómo se modifican estos resultados si el trabajo realizado por el alumno se vincula con la carrera que cursa. Para investigarla en primer lugar se incluye la variable binaria "trabajo vinculado con la carrera" y en otra aproximación, se estima separadamente el modelo sólo para los alumnos que trabajan en temas vinculados y sólo para quienes trabajan en temas no vinculados.

Como se observa en la segunda columna de la tabla 4.3, adicionando al modelo anterior un control por trabajo vinculado con la carrera, se encuentra que la relación del rendimiento con las horas trabajadas y su cuadrado se mantiene positiva y decreciente. Sin embargo, es interesante notar que el coeficiente de las horas trabajadas es menor que en el modelo precedente, por lo que el tiempo dedicado al trabajo por semana que maximiza el rendimiento se reduce de 14.5 a 10 horas semanales. Esta diferencia sugiere que el coeficiente de la estimación anterior podría resultar sobreestimado al no controlar por el efecto de horas trabajadas en los trabajos vinculados a la carrera.

Considerando que las estimaciones anteriores pueden estar sesgadas por la potencial autoselección de los alumnos de mayor rendimiento en trabajos vinculados con la carrera de estudio, en las dos últimas columnas de la tabla 4.3 se presentan estimaciones del modelo de rendimiento tomando por separado a los alumnos que trabajan en temas vinculados y a los que tienen trabajos no vinculados con su carrera.

\section{Relación no lineal horas de trabajo vinculado-rendimiento}

En los resultados de la tabla 4.3 para el grupo que trabaja en temas vinculados con la carrera se encuentra que el coeficiente para las horas trabajadas es significativo y positivo, mientras que, nuevamente la relación marginal es además decreciente, de acuerdo con el coeficiente negativo y significativo para el cuadrado de las horas trabajadas.

Sin embargo, es interesante encontrar que si bien la relación es decreciente, el hecho de que el trabajo esté vinculado con la carrera extiende los efectos positivos del trabajo hasta 24.5 horas trabajadas semanales. A partir de esa cantidad de horas, aún cuando el trabajo esté vinculado a la carrera, el efecto de la restricción temporal domina al de la complementariedad del trabajo con el estudio. En comparación con los resultados previos, los efectos positivos del trabajo se mantienen aún trabajando hasta 14.5 horas semanales más en promedio, si el trabajo está vinculado con la carrera. 
Este resultado se relaciona con los argumentos de que el aprendizaje se desarrolla mejor practicando lo que se estudia y con las estadísticas presentadas en la Subsección III.4.

\section{Relación no lineal horas de trabajo no vinculado-rendimiento}

En contraste, los resultados de las estimaciones para el grupo que trabaja en temas no vinculados con la carrera reflejan una relación negativa entre las horas trabajadas y el rendimiento. Esto indica que para cualquier nivel de horas trabajadas, el tiempo dedicado a trabajos no vinculados se asocia negativamente con el rendimiento.

Uno de los resultados de esta parte del trabajo es que cuando se incluye sólo la variable horas trabajadas en el modelo, se ignora que puede existir un efecto diferencial sobre el rendimiento de acuerdo al tiempo que se trabaje. Esto es, se observa una relación no lineal. En general, el trabajo del estudiante puede ser beneficioso para su rendimiento, siempre que trabaje una moderada cantidad de horas. Esa relación alcanza un máximo a partir del cual las horas trabajadas se relacionan negativamente con el rendimiento. Lo cual puede explicarse a través de la restricción temporal del estudiante, dado que las horas trabajadas restan tiempo disponible para realizar tareas como estudiar. ${ }^{33}$

Por último, considerando sólo los alumnos con trabajo vinculado, se observa esa relación curvilínea, y que el trabajo complementario tiene efectos positivos para un intervalo mayor de horas trabajadas. Pero igualmente, existe un efecto negativo más allá de ese máximo, dada la dotación de tiempo disponible del estudiante, aún cuando el trabajo sea complementario a la carrera. En contraste, considerando sólo al grupo con trabajo no vinculado, las horas de trabajo no vinculado se relacionan negativamente con el desempeño para todos los niveles de horas trabajadas.

En definitiva, pareciera que las condiciones deseables para que el trabajo del estudiante no afecte el desempeño, e incluso pueda mejorarlo son: que el trabajo se vincule con los temas de estudio, y que éste no exceda de 5 horas diarias de dedicación.

\section{Conclusiones y líneas de investigación futura}

En este trabajo se fomula una serie de preguntas sobre la relación entre el tiempo dedicado al trabajo y el rendimiento académico de estudiantes universitarios. Para aproximar respuestas se utilizan datos del Primer censo de estudiantes de universidades nacionales desarrollado en 1994.

En primer lugar se encuentra que existen diferencias sustanciales entre estimar la relación trabajo-rendimiento a través de una variable binaria "trabaja" o estimarla con un indicador del tiempo dedicado a trabajar por medio de la variable "horas trabajadas por semana". En el primer caso la relación resulta positiva y en el segundo es negativa, sugiriendo que el coeficiente de la variable "trabaja" subestima los efectos negativos del tiempo dedicado a trabajar; y puede incluso captar otros factores, como por ejemplo los inobservables relacionados con la decisión de trabajar.

En segundo lugar, en las estimaciones para el conjunto de alumnos que trabajan, se encuentra un resultado que compatibiliza con los argumentos contrapuestos acerca de la relación entre las horas trabajadas y el rendimiento. Con una especificación lineal, se encuentra que las horas trabajadas se relacionan negativamente con el rendimiento, tanto para trabajos vinculados como no vinculados. Mientras que si se postula una especificación no lineal, las horas trabajadas se asocian en forma positiva y decreciente con el rendimiento. En otros términos, en un tramo de baja cantidad de

33 Estos resultados se mantienen por Mínimos cuadrados en dos etapas. También se realizaron estimaciones por intervalos de horas trabajadas, pero, al reducirse las muestras, los efectos no resultan significativos en todos los tramos. En cuanto a las estimaciones realizadas por distintos agrupamienos de carreras, por ejemplo, por rama-disciplina, se encuentran los mismos signos para 10 de las 17 ramas-disciplinas. 
horas el rendimiento del alumno puede llegar a ser maximizado (14.5 horas semanales); más allá de ese punto las horas trabajadas inciden negativamente.

Si se considera sólo el efecto de las horas trabajadas en temas vinculados, se encuentra que la relación no lineal se mantiene y que el estudiante puede aprovechar los beneficios del trabajo complementario hasta una mayor cantidad de horas trabajadas (24.5 horas semanales). En contraste, si el trabajo del estudiante no se vincula, la relación entre las horas trabajadas y el rendimiento es negativa, aún cuando trabaje pocas horas.

En conclusión, podría decirse que: 1) La incidencia positiva del trabajo del estudiante se manifiesta sólo cuando el trabajo implica una limitada dedicación horaria y un cierto grado de vinculación con los temas de la carrera que estudia. 2) Los trabajos sin vinculación con los temas de estudio afectan negativamente el rendimiento académico del alumno, cualquiera sea el nivel de horas trabajadas. 3) Un alumno que trabaje en temas vinculados con la carrera puede aumentar su rendimiento trabajando una moderada cantidad de horas semanales (hasta 5 horas diarias).

Resumiendo, en términos de desempeño académico, los trabajos compatibles con la etapa de estudiante del alumno son aquellos de baja dedicación horaria y relacionados con los contenidos de estudio.

Los resultados encontrados sobre el tipo de trabajo del estudiante que preserva su rendimiento académico coinciden con las condiciones del trabajo del estudiante previstas en la Ley de Pasantías. En su artículo 11 establece que "Las pasantías se extenderán durante un mínimo de dos meses y un máximo de un año, on una adtividad semanal no mayor de cinoo días en cuyo transcurso el pasante amplirá jornadas de hasta cuatro horas de labor" .

En este sentido, este trabajo contribuye a aportar fundamentos empíricos a lo establecido en la legislación universitaria sobre las pautas de compatibilidad del trabajo con el desarrollo de las actividades académicas. Incluso encuentra que para niveles moderados de horas trabajadas este tipo de práctica vinculada, además de ser positiva para el futuro desempeño profesional, podría ser beneficiosa también para el desempeño académico del alumno. Asimismo, revela que los alumnos que trabajen más de las horas límite 0 en trabajos no vinculados, dadas otras características, tienden a estar en desventaja respecto al resto de los estudiantes universitarios en términos de rendimiento académico.

En el trabajo se reconoce que los resultados obtenidos deben verse como un primer paso necesario para extender el análisis en varias dimensiones para versiones futuras. Por un lado, avanzar en el desarrollo teórico del problema planteado. Por otro lado, se deja para nuevas versiones perfeccionar los avances realizados con esta muestra para controlar el problema de heterogeneidad entre carreras y cohortes y la utilización de una muestra que permita controlar el problema de endogeneidad por el método de Variables instrumentales.

No obstante, las preguntas que se formulan y las respuestas que se ofrecen a partir del estudio de los datos del Censo de estudiantes de universidades nacionales de 1994 constituyen un aporte para el conocimiento de la relación trabajo-rendimiento estudiantil, dado un conjunto de factores explicativos. 


\section{Referencias}

Albarracín Sanchez, D. (2004): “Usos del empleo a tiempo parcial en Europa y relaciones de género". Fundación CIREM. (mimeo).

Carr, R, J. Wright y C. J. Brody (1996): "Effects of high school work experience a decade later; Evidence from the National Longitudinal Survey". Sociology of E ducation 69, 66-81

Coleman, J. S. (1961): The adolesœent society, G lencoe, IL:Free Press.

Coleman, J. S., E. Campbell, C. Hobson, J. McPartland, A. Mood, F. Weinfeld y R. York. (1966): E quality of educational opportunity, Washington DC: US G overnment printing office.

D 'Amico , R. (1984): “ D oes employment during High School impair academic progress?” Sociology of E ducation, 57, 152-64.

Di Gresia, L., A. Porto y L. Ripani y W. Sosa Escudero (2003): "Rendimiento de los Estudiantes de las Universidades Públicas Argentinas". A nales de las 36as Jornadas Internacionales de Finanzas Públicas. Universidad Nacional de Córdoba.

Di Gresia, L., M. Fazio, A. Porto, L. Ripani y W. Sosa Escudero (2004): "Rendimiento y Productividad de los Estudiantes. El Caso de las Universidades Públicas Argentinas", Departamento de Economía, Facultad de Ciencias Económicas, Universidad Nacional de La Plata. (mimeo).

Di Gresia, L. y A. Porto (2004): "Dinámica del desempeño académico". D ocumento de Trabajo $\mathrm{N}^{\circ} 49$, D epartamento de Economía, Facultad de Ciencias Económicas, Universidad Nacional de La Plata.

Eckstein, Z. y K. Wolpin (1997): "Youth employment and academic performance in High School", CEPR Discussion paper.

Ehrenberg, R. y D. Sherman (1987): "Employment while in College, academic achievement and postcollege outcomes", The Journal of H uman Resourœe, 22 (1), 1-23.

Gertel, H. (1999): "Los estudiantes de la educación superior en la Argentina: Un análisis empírico de su localización, campo profesional y características familiares en 1998", (mimeo).

Giovagnoli, P. (2002): “D eterminantes de la deserción y graduación universitaria: una aplicación utilizando modelos de duración", Documento de trabajo N³7. Departamento de Economía, Facultad de Ciencias Económicas, Universidad Nacional de La Plata.

Greenberger y Steinberg (1986): W hen teenagers work: the psyoological and social oosts of adolescent employment. New York: Basic books.

Hanushek, E. (1993): "Can equity be separated from efficiency in school finance debate?, en Hanushek, E. (1997): "Assessing the effects of school resources on student performance: an update". E ducational E valuation and Policy A nalysis, 19, 141-164.

Hanuskek, E. S. Rivkin y L. Taylor (1996): "The identification of school resource effects", E ducational E conomics, 4 (2), 105-125.

Hanushek, E. (2003): "The failure of input-based schooling policies", E conomic Journal, 113, 64-98.

Holland, A. y T. Andre (1987): " Participation in extracurricular activities in secondary school: what is known, what needs to be known?, R eview of $\mathrm{E}$ ducational R esearch, 57, 437-466.

Hood, A, A. Craig y B. Ferguson (1992): " The impact of athletics, part-time employment and other academic activities on academic achievement", Journal of C ollege Student D evelopment, 33, 447-453.

Lillydahl, J. (1990): "Academic achievement and part-time employment of High school students", Journal of E conomic E ducation, 21 (3), 307-316.

Llach, J., S. Montoya y F. Roldán. (2000): E ducación para todos. IERAL

McGuckin, R. y D. Winkler (1979): "University Resources in the Production of Education", The Review of E conomics and Statistics, 61 (2), 242-248.

Ministerio de Cultura y Educación de la Nación: Ley 25165/ 99 de Pasantías y Ley 24521/95 de Educación Superior.

Ministerio de Cultura y Educación de la Nación: Reglamento G eneral de Becas Universitarias. 
NCES (2002): "The persistence of employees who pursue postsecondary study", en Stats in Brief, NCES 2002-118, US D epartment of Education.

Paul, H. (1982): "The impact of outside employment on student academic achievement in Macroeconomic principles", Journal of E conomic E ducation, 13, 51-56.

Pauly, E., H. Kopp y J. Haimson (1995): H ome grown lessons, innovative programs linking school and work. Jossey-Bass. San Francisco.

Porto, A. y L. Ripani (2003): “Algunos datos sobre la educación universitaria en la Argentina” D epartamento de Economía. Facultad de Ciencias Económicas, UNLP. (mimeo).

Pritchett L. y D. Filmer (1997): "What education production functions really show". Policy Research working paper 1795. Washington D C: The World Bank.

Ruhm, C. (1997): “Is High school employment consumption or investment?”, Journal of L abor E conomics, 15 (4), 735-776.

Schill, W., R. McCartin y K. Meyer (1985): "Youth employment: its relationship to academic and family variables", Journal of V ocational Behaviour, 26, 155-163.

Secretaría de Políticas Universitarias: Anuario estadístico 1998 y 1999-2000.

Stern, D. , N. Finkelstein, M. Urquiola y H. Cagampang (1997): "What difference does it make if school and work are connected? Evidence on Co-operative education in the United States" E conomics of E ducation Review, 16 (3), 213-229.

Stinebrickner, T. y R. Stinebrickner (2003): "Working during school and academic performance", Journal of L abor E conomics, 21 (2), 473-491.

Stone, J., D. Stern, C. Hopkins y M. McMillon (1990): “ Adolescents perceptions of their work: school supervised and non-school-supervised" Journal of V ocational E ducation Research, 15, 31-53.

Summers, A. y B. Wolfe (1977): “D o schools make a difference?” The A merican E conomic Review, 67, 639-652.

Turner, M. (1994): "The effects of part-time work on High school students academic achievement". University of Maryland. (mimeo)

Tyler J. (2003): "Using state child labor laws to identify the effect of school-year work on High school achievement". Journal of L abor E conomics, 21 (2), 381-408. 


\section{Anexo 1}

\section{Descripción de Variables explicativas utilizadas}

\begin{tabular}{|c|c|}
\hline Variable & 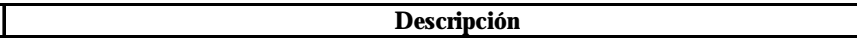 \\
\hline Rendimiento & Total de materias aprobadas/ años desde el ingreso \\
\hline Edad & Edad en años cumplidos al 1/ 9/94 \\
\hline Sexo & Variable binaria: 1 varón, 0 mujer \\
\hline Nacionalidad & V ariable binaria: 1 extranjero, 0 argentino \\
\hline Estado civil: unido & Variable binaria: 1 unido, 0 otra categoría \\
\hline Estado civil: casado & V ariable binaria: 1 casado, 0 otra categoría \\
\hline Estado civil: separado & Variable binaria: 1 separado, 0 otra categoría \\
\hline Estado civil: divorciado & Variable binaria: 1 divorciado, 0 otra categoría \\
\hline Estado civil: viudo & V ariable binaria: 1 viudo, 0 otra categoría \\
\hline Estado civil: soltero (base) & V ariable binaria: 1 soltero, 0 otra categoría \\
\hline Escuela secundaria privada & Variable binaria: 1 privada, 0 pública \\
\hline Residencia durante el período de clases & V ariable binaria: 1 otra, 0 misma \\
\hline Cambio de residencia a causa de los estudios & V ariable binaria: $1 \mathrm{si}, 0 \mathrm{no}$ \\
\hline Años desde el ingreso & Año 1995 menos el año de ingreso \\
\hline Edad al ingreso & Edad en años cumplidos al ingresar en la unidad académica \\
\hline Horas de estudio semanales (con clases) & Cantidad de horas semanales dedicadas, en promedio, al estudio, con la asistencia a clases \\
\hline Años de educación del padre & Número de años de instrucción \\
\hline Años de educación de la madre & Número de años de instrucción \\
\hline Fuente de ingresos: trabajo personal & Variable binaria: 1 trabajo personal, 0 otra categoría \\
\hline Fuente de ingresos: aporte familiar & V ariable binaria: 1 aporte familiar, 0 otra categoría \\
\hline Fuente de ingresos: trabajo personal y aporte familiar & Variable binaria: 1 trabajo personal y aporte familiar, 0 otra categoría \\
\hline Fuente de ingresos: sólo beca de estudio & Variable binaria: 1 beca de estudio, 0 otra categoría \\
\hline Fuente de ingresos: beca de estudio y aporte familiar & V ariable binaria: 1 beca de estudio y aporte familiar, 0 otra categoría \\
\hline Fuente de ingresos: otras & Variable binaria: 1 otras, 0 otra categoría \\
\hline Trabajo & Variable binaria: 1 trabaja, 0 no \\
\hline Horas trabajadas por semana & Promedio de horas semanales dedicadas al trabajo \\
\hline Trabajo vinculado con la carrera & V ariable binaria: 1 vinculado, 0 no \\
\hline Categoría ocupacional: patrón con 5 empleados & Variable binaria: 1 patrón de 5,0 otra categoría \\
\hline Categoría ocupacional: patrón con 6 empleados & Variable binaria: 1 patrón de 6, 0 otra categoría \\
\hline Categoría ocupacional: empleado sector privado & Variable binaria: 1 empleado sector privado, 0 otra categoría \\
\hline Categoría ocupacional: empleado sector público & V ariable binaria: 1 empleado sector público, 0 otra categoría \\
\hline Categoría ocupacional: profesional univ. Cta. propia & Variable binaria: 1profesional, 0 otra categoría \\
\hline Categoría ocupacional: Cta. propia no profesional & Variable binaria: 1 no profesional, 0 otra categoría \\
\hline Empleado servicio doméstico/ trabajo familiar & V ariable binaria: 1 empleado doméstico/ trabajador fliar, 0 otra categoría \\
\hline Gasto por alumno promedio en la universidad & Gasto total sobre número de alumnos para cada universidad \\
\hline Composición promedio de cuerpo docente en la universidad & $\begin{array}{l}\text { Nrodocentes equivalentes con dedicación semi y exclusiva dividido el nro docentes con } \\
\text { dedicación simple }\end{array}$ \\
\hline Cantidad de docentes equivalentes en la universidad & Suma ponderada de las diferentes categoría de docentes \\
\hline
\end{tabular}

En base al Censo de Estudiantes de universidades nacionales (1994). 


\section{Anexo 2}

\section{Categońas de Ramas y Disciplinas de estudio ${ }^{34}$}

\section{Ciencias Básicas y Tecnológicas.}

1.1. Ciencias Agropecuarias.

1.2. Arquitectura.

1.3. Ingeniería, Agrimensura y Tecnología.

1.4. Ciencias Exactas y Naturales.

1.5. Bioquímica, Farmacia y Química.

\section{Ciencias Sociales.}

2.1. Administración, Ciencias Económicas y Organización.

2.2. Derecho, Ciencias Públicas y Diplomáticas.

2.3. O tras Ciencias Sociales.

\section{Humanidades.}

3.1. Filosofía y Letras.

3.2. Ciencias de la Educación.

3.3. O tras Ciencias Humanas.

3.4. Bellas Artes y Música.

\section{Ciencias Médicas.}

4.1. Medicina.

4.2. O dontología.

4.3. Paramédicas.

4.4. Auxiliares de la Medicina.

34 Anuario 99-00 de Estadísticas Universitarias, Secretaría de Políticas Universitarias. 